\title{
Seshadri positive submanifolds of polarized manifolds*
}

\author{
Lucian Bădescu and Mauro C. Beltrametti
}

\begin{abstract}
Let $Y$ be a submanifold of dimension $y$ of a polarized complex manifold $(X, A)$ of dimension $k \geq 3$, with $1 \leq y \leq k-1$. We define and study two positivity conditions on $Y$ in $(X, A)$, called Seshadri $A$-bigness and (a stronger one) Seshadri $A$-ampleness. In this way we get the natural generalization of the theory initiated by Paoletti in 28] (which corresponds to the case $(k, y)=(3,1))$ and subsequently generalized and completed in 5 . (regarding curves in a polarized manifold of arbitrary dimension). The theory presented here, which is new even if $y=k-1$, is motivated by a reasonably large area of examples.
\end{abstract}

\section{Introduction}

Let $(X, A)$ be a smooth complex polarized variety (manifold) of dimension $k \geq 3$, and let $Y$ be a smooth (connected) subvariety of dimension $y \geq 1$. Let $X_{Y}$ be the variety obtained from $X$ by blowing up $Y$, let $\pi: X_{Y} \rightarrow X$ be the canonical morphism and let $E=\pi^{-1}(Y)$ be the exceptional divisor of $\pi$. Let $N$ denote the normal bundle of $Y$ in $X$. Then one can define the Seshadri constant $\varepsilon(Y, A)$ of $Y$ with respect to the polarization $A$ as

$$
\varepsilon(Y, A):=\sup \left\{\eta \in \mathbb{Q} \mid A^{*}-\eta E \text { is ample }\right\},
$$

where $A^{*}=\pi^{*}(A)$. As $A$ is ample on $X$ and the line bundle $\mathcal{O}_{X_{Y}}(-E)$ is $\pi$-ample (where $\pi: X_{Y} \rightarrow X$ is the structural morphism of $X_{Y}$ ), this definition makes sense and yields the inequality $\varepsilon(Y, A)>0$. Then $\varepsilon(Y, A)$ is a strictly positive real number. Motivated by the study of the gonality of curves and by the behavior of restriction of the stable vector bundles (see [29]), the Seshadri constant $\varepsilon(Y, A)$ was used by Paoletti in 28] to study the so-called Seshadri positive curves in a polarized threefold. Subsequently, this theory was generalized and completed to the case when $Y$ is a smooth curve in a smooth polarized variety $(X, A)$ of arbitrary dimension $k \geq 3$ in [5. Specifically, for every $\eta \in(0, \varepsilon(Y, A))$ define the numerical invariant

$$
\delta_{\eta}(Y, A):=\eta^{k-3}(\eta \operatorname{deg}(N)-(k-2) d),
$$

where $d$ is the degree of $Y$ with respect to the polarization $A$. In this case $Y$ is said to be Seshadri $A$-big in $(X, A)$ if there is $\eta \in(0, \varepsilon(Y, A))$ such that $\delta_{\eta}(Y, A)>0$. This definition has a natural geometrical interpretation given by Lemma 1.9 below.

Moreover, at the end of [5] it was left open the problem of finding the natural general setting for a theory of Seshadri positivity for a submanifold $Y$ of any dimension $y \geq 1$ in a complex polarized manifold of dimension $k \geq 3$, which should generalize the case of Seshadri positive curves in a polarized manifold. And a possible such definition of Seshadri positivity was suggested in the last section of [5], but, unfortunately, it turned out to be much too strong to work with if $y \geq 2$.

*2010 Mathematics Subject Classification. Primary 14E25, 14C25; Secondary 14D15, 14F20.

Keywords and phrases. Seshadri constant, Seshadri $A$-big, Seshadri $A$-ample, variety defined in a given degree, formal rational functions, cohomological dimension 
The aim of the present paper is to give, in our opinion, the natural generalization of the concept of Seshadri positivity to submanifolds of dimension $y \geq 1$ in a smooth polarized manifold $(X, A)$ and to recuperate the main results (proved in [28] and [5] in the case when $Y$ is a curve) in general. To this end, for every submanifold $Y$ of dimension $y \geq 1$ of a polarized manifold $(X, A)$ of dimension $k \geq 3$ and for every $\eta \in(0, \varepsilon(Y, A))$, define the numerical invariant

$$
\delta_{\eta}(Y, A):=-\sum_{t=0}^{k-2}\left(\begin{array}{c}
k-2 \\
t
\end{array}\right) \eta^{t} \int_{Y} s_{y-k+t+2}(N) \cdot c_{1}\left(A_{Y}\right)^{\cdot(k-t-2)},
$$

where $s_{i}(N)$ is the $i$-th Segre class $N, c_{1}\left(A_{Y}\right)$ is the first Chern class of the restriction of $A$ to $Y$, and $\int_{Y} \alpha$ denotes the degree of a class of a zero-cycle $\alpha$ on $Y$. Thus the coefficients of the polynomial function given by $\eta \mapsto \delta_{\eta}(Y, A)$ depend on the invariants of the closed embedding of $Y$ in the polarized manifold $(X, A)$. It turns out that the polynomial function defined by (2) is the natural generalization of (11). Now, in general, we say that $Y$ is Seshadri $A$-big if there exists an $\eta \in(0, \varepsilon(Y, A))$ such that $\delta_{\eta}(Y, A)>0$. This general definition is geometrically motivated by Lemma 1.9 again. Moreover, starting with this definition one can provide a reasonably large area of interesting examples of Seshadri $A$-big manifolds in any dimension and codimension, and one can prove most of the results contained in [28 and [5] in this general setting. It is worth noting that the theory of Seshadri positivity of a submanifold $Y$ of a polarized manifold $(X, A)$ presented here is of interest even when $Y$ is a hypersurface of $X$ (see Section 2).

The paper is organized as follows. In Section 10 we give the basic definitions of Seshadri $A$-bigness and Seshadri $A$-ampleness for a submanifold $Y$ of dimension $y \geq 2$ of a complex polarized projective manifold $(X, A)$ (see Definition [1.10), we prove some preliminary results and we give a few examples of how to compute the Seshadri constant $\varepsilon(Y, A)$.

In Section 2 we discuss the case when $\operatorname{codim}_{X}(Y)=1$. In this case there are surprisingly many examples. Specifically, we first prove that if the normal bundle $N$ of $Y$ in $X$ is ample then $Y$ is Seshadri $A$-big in $X$. Then we show that the same conclusion holds under a much weaker hypothesis (Corollary 2.3) and we give three relevant examples in which $N$ is not ample but satisfies that weaker hypothesis.

Section 3 is in some sense the "core" of the paper and deals with the case of submanifolds of codimension $\geq 2$, in which case the concepts of Seshadri $A$-bigness and $A$-ampleness become rather strong. Indeed in this section the task is to provide interesting examples of submanifolds $Y$ of a polarized manifold $(X, A)$ of codimension $\geq 2$ which are Seshadri $A$-big (in fact, Seshadri $A$-ample by the last statement of Corollary 5.3 . This gives strong motivations for the positivity notions introduced in Section 1 in arbitrary codimension. We also point out in paragraph 3.6 a useful expression which allows us to compute Chern classes for manifolds in projective space. Let us overview the examples we have. As a consequence of a more general result, we prove that, if we fix a projective embedding $X \hookrightarrow \mathbb{P}^{n}$, every smooth complete intersection in $X$ of codimension 2 is $\mathcal{O}_{X}(1)$-ample (see Propositions 3.4 and 3.5). We also show that the Veronese surface $Y$ in $\mathbb{P}^{5}$ is Seshadri $\mathcal{O}_{\mathbb{P}^{5}}(1)$-ample, as well as its projection from a general point of $\mathbb{P}^{5}$ is Seshadri $\mathcal{O}_{\mathbb{P}^{4}}(1)$-ample. Further relevant examples of subvarieties of $\mathbb{P}^{k}$ that are Seshadri $\mathcal{O}_{\mathbb{P} k}(1)$-ample are given by some rational normal scrolls of dimension 2, and some Segre embeddings (Proposition 3.8). Another example of a Seshadri $A$-big surface in $\mathbb{P}^{5}$ is given by the geometrically ruled surface $Y=C \times \mathbb{P}^{1}$, with $C \subset \mathbb{P}^{2}$ a smooth elliptic curve, embedded in $\mathbb{P}^{5}$ via the Segre embedding $\mathbb{P}^{2} \times \mathbb{P}^{1} \hookrightarrow \mathbb{P}^{5}$ (Proposition 3.15). This latter example has some special interest because the surface in question is not rational. Finally, Proposition 3.16 (noticed in Paoletti [28] in the case $(k, y)=(3,1))$ is important because it allows to construct many more examples of Seshadri $A$-big (respectively, Seshadri $A$-ample) submanifolds of a polarized manifolds $(X, A)$, starting from some already known ones.

In last two sections we show that the definitions given in Section 2 and the examples of Section 3 offer the "correct setting" for the study of submanifolds of dimension $\geq 2$ in a 
polarized manifold $(X, A)$ which are Seshadri positive. In Section 4 we show how the Seshadri positivity of a submanifold $Y \subset X$ is related to the theory of formal-rational functions of $X$ along $Y$, as well as to the cohomological dimension of the complement $X \backslash Y$. Precisely, if $Y$ is Seshadri $A$-big (respectively, Seshadri $A$-ample), then $Y$ is G2 (respectively G3) in $X$ in the sense of Hironaka-Matsumura 24. This allows us to give some estimates for the cohomolgical dimension of $X \backslash Y$ (see Theorem 4.5).

In Section 5 a criterion to distinguish between Seshadri $A$-ampleness and Seshadri $A$-bigness is proved (see Theorem [5.1, compare with [5, Theorem 3.1]). This criterion asserts that a Seshadri $A$-big subvariety $Y$ is $A$-ample in $X$ if and only if any irreducible hypersurface in $X$ intersects $Y$. Moreover, we show that if $Y$ is Seshadri $A$-big, the number of all hypersurfaces of $X$ not meeting $Y$ is finite. As a consequence of Theorem 5.1 we can provide explicit examples of submanifolds $Y \subset X$ which are Seshadri $A$-big, but not Seshadri $A$-ample (see Example 5.5). The section ends up with some further examples, comments and remarks.

Unless otherwise stated, the terminology and the notation used throughout are standard. As far as the Chern and the Segre classes computations are concerned (see Sections 1 and 3) we shall follow the notation, conventions and some basic results of Fulton's book 12. All algebraic varieties (or schemes) will be defined over the field $\mathbb{C}$ of complex numbers.

Dedication. In the middle nineties (inspired by [28]) we started studying these kind of problems for curves in a polarized manifold of arbitrary dimension in [5], together with our colleague and friend Paolo Francia (who passed away in July 2000). We want to dedicate this paper to his memory.

\section{Basic definitions and preliminary results and examples}

Let $X$ be a smooth complex manifold of dimension $k \geq 3$ and let $Y \subset X$ be a smooth subvariety of $X$ of dimension $y$ such that $1 \leq y \leq k-2$. Let $N:=N_{Y \mid X}$ be the normal bundle of $Y$ in $X$. Then $N$ is a rank $k-y$ vector bundle on $Y$. Let

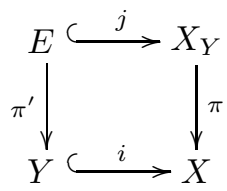

be the blowing up of $X$ along $Y$, with $E:=\pi^{-1}(Y)$ the exceptional divisor and $i$ and $j$ the natural closed immersions. Then $E=\mathbb{P}\left(N^{\vee}\right)$, where $N^{\vee}$ is the dual of $N$. We are using the notation of Grothendieck, i.e., if $\mathcal{F}$ is a vector bundle on an algebraic variety $Z$, we denote by $\mathbb{P}(\mathcal{F})$ the projective bundle of hyperplanes of $\mathcal{F}$ and by $\mathcal{O}_{\mathbb{P}(\mathcal{F})}(1)$ is the tautological line bundle of $\mathbb{P}(\mathcal{F})$. For any ample line bundle $A$ on $X$, set $A^{*}:=\pi^{*}(A)$ and let $d:=Y \cdot A^{\cdot y}$ be the degree of $Y$ with respect to $A$.

According to Fulton [12, (3.1), (B.5.5)], if $\mathcal{E}$ is a vector bundle on an algebraic variety $Z$, define the Segre class $s_{j}(\mathcal{E})$ of order $j$ of $\mathcal{E}$ by

$$
s_{j}(\mathcal{E}):=\pi_{*}\left(c_{1}\left(\mathcal{O}_{\mathbb{P}\left(\mathcal{E}^{\vee}\right)}(1)\right)^{\cdot(\operatorname{rank}(\mathcal{E})-1+j)}\right),
$$

where $\pi: \mathbb{P}\left(\mathcal{E}^{\vee}\right) \rightarrow Z$ is the canonical projection. Then $s_{j}(\mathcal{E})$ is the rational equivalence class of a cycle of codimension $j$ in $Z$. In particular, $s_{0}(\mathcal{E})=1$ and $s_{j}(\varepsilon)=0$ if $j<0$ or $j>\operatorname{dim}(Z)$. Moreover, if $\operatorname{rank}(\mathcal{E})=1$ then $\mathbb{P}\left(\mathcal{E}^{\vee}\right)=\mathbb{P}\left(\mathcal{E}^{-1}\right)=Z, \pi=\operatorname{id}_{Z}$ and $\mathcal{O}_{\mathbb{P}\left(\mathcal{E}^{\vee}\right)}(1)=\mathcal{E}^{-1}$. In particular, if $\mathcal{E}$ is invertible it follows that $s_{j}(\mathcal{E})=(-1)^{j} c_{1}(\mathcal{E})^{\cdot j}$ for every $j \geq 0$.

First we need the following: 
Lemma 1.1 With the notation as in diagram (3), set $A_{Y}:=A \otimes \mathcal{O}_{Y}$. Then for every nonnegative integer $r$ we have the following equalities:

$$
\int_{X_{Y}} E^{\cdot(k-r)} \cdot c_{1}\left(A^{*}\right)^{\cdot r}= \begin{cases}(-1)^{k-r-1} \int_{Y} s_{y-r}(N) \cdot c_{1}\left(A_{Y}\right)^{\cdot r} & \text { if } r \leq y, \\ 0 & \text { if } r \geq y+1 .\end{cases}
$$

Proof. One has $\mathcal{O}_{X_{Y}}(-E)_{E}=\mathcal{O}_{\mathbb{P}\left(N^{\vee}\right)}(1)=\mathcal{O}_{E}(1)$. Therefore we get the following equalities of $r$-cycles (modulo the rational equivalence)

$$
\begin{aligned}
E^{\cdot(k-r)} & =E_{E} \cdots E_{E}=c_{1}\left(\mathcal{O}_{E}(-1)\right) \cdots c_{1}\left(\mathcal{O}_{E}(-1)\right) \quad(k-r-1 \text { times }) \\
& =(-1)^{k-r-1} c_{1}\left(\mathcal{O}_{\mathbb{P}\left(N^{\vee}\right)}(1)\right)^{\cdot(k-r-1)} .
\end{aligned}
$$

Thus, by using the projection formula ([12, Proposition 2.5, (c)]) and expression (44), we get

$$
\begin{aligned}
\int_{X_{Y}} E^{\cdot(k-r)} \cdot c_{1}\left(A^{*}\right)^{\cdot r} & =(-1)^{k-r-1} \int_{X_{Y}} c_{1}\left(\mathcal{O}_{\mathbb{P}\left(N^{\vee}\right)}(1)\right)^{\cdot(k-r-1)} \cdot c_{1}\left(A^{*}\right)^{\cdot r} \\
& =(-1)^{k-r-1} \int_{X} \pi_{*}\left(c_{1}\left(\mathcal{O}_{\mathbb{P}\left(N^{\vee}\right)}(1)\right)^{\cdot(k-r-1)}\right) \cdot c_{1}(A)^{\cdot r} \\
& \left.=(-1)^{k-r-1} \int_{Y} \pi_{*}^{\prime}\left(\mathcal{O}_{\mathbb{P}\left(N^{\vee}\right)}(1)\right)^{\cdot(k-r-1)}\right) \cdot c_{1}\left(A_{Y}\right)^{\cdot r} \\
& =(-1)^{k-r-1} \int_{Y} s_{y-r}(N) \cdot c_{1}\left(A_{Y}\right)^{\cdot r} \cdot
\end{aligned}
$$

Finally, if $r \geq y+1$ then $y-r<0$, whence $s_{y-r}(N)=0$.

Definition 1.2 (Paoletti [28]) Let $X$ be a smooth complex manifold of dimension $k \geq 3$, let $Y \subset X$ be a smooth subvariety of $X$ of positive dimension $\operatorname{dim}(Y):=y \leq k-2$, and fix an ample line bundle $A$ on $X$. Define the Seshadri constant of $Y$ with respect to $A$ as

$$
\varepsilon(Y, A):=\sup \left\{\eta \in \mathbb{Q} \mid A^{*}-\eta E \text { is ample }\right\} .
$$

We claim that $0<\varepsilon(Y, A)<\infty$. Indeed, since $A$ is ample on $X$ and $\mathcal{O}_{X_{Y}}(-E)$ is $\pi$-ample on $X_{Y}$, by [17, II (4.6.13), (ii)], we know that $A^{*}-\frac{1}{n} E$ is ample for $n \gg 0$, whence $\varepsilon(Y, A)>0$. On the other hand, for every ample divisor $H$ on $X_{Y}$ one has $H^{k-1} \cdot\left(A^{*}-\eta E\right)<0$ for $\eta \gg 0$, so that $\varepsilon(Y, A)<\infty$.

Define also the numerical invariant $\delta(Y, A)$ by

$$
\delta(Y, A):=-\sum_{t=0}^{k-2}\left(\begin{array}{c}
k-2 \\
t
\end{array}\right) \varepsilon(Y, A)^{t} \int_{Y} s_{y-k+t+2}(N) \cdot c_{1}\left(A_{Y}\right)^{\cdot(k-t-2)} .
$$

Moreover, for every $\eta \in(0, \varepsilon(Y, A))$ also define

$$
\delta_{\eta}(Y, A):=-\sum_{t=0}^{k-2}\left(\begin{array}{c}
k-2 \\
t
\end{array}\right) \eta^{t} \int_{Y} s_{y-k+t+2}(N) \cdot c_{1}\left(A_{Y}\right)^{\cdot(k-t-2)} .
$$

The numerical invariants $\varepsilon(Y, A), \delta(Y, A)$ and $\delta_{\eta}(Y, A)$ will play an important role in the studying the embedding $i: Y \hookrightarrow X$. For instance the numerical invariant $\delta_{\eta}(Y, A)$ will be motivated by Lemma 1.9 below. From the definitions it follows immediately that

$$
\delta(Y, A)>0 \Longrightarrow \delta_{\eta}(Y, A)>0 \text { for every } \eta \in(0, \varepsilon(Y, A)) \text { which is close to } \varepsilon(Y, A) .
$$

Moreover, if $\delta(Y, A)=0$ and if $f^{\prime}(\varepsilon(Y, A))<0$ then $\delta_{\eta}(Y, A)>0$ for every $\eta \in(0, \varepsilon(Y, A))$ which is close to $\varepsilon(Y, A)$, where $f: \mathbb{R} \rightarrow \mathbb{R}$ is the polynomial function defined by $f(\eta)=\delta_{\eta}(Y, A)$ for all $\eta \in \mathbb{R}$, and $f^{\prime}$ denotes the derivative of $f$. 
Remark 1.3 From the definition of the Seshadri constant it follows that $\varepsilon\left(Y, A^{\otimes s}\right)=s \varepsilon(Y, A)$, for every integer $s \geq 1$. In particular, for every $\eta \in(0, \varepsilon(Y, A))$ we have $s \eta \in\left(0, \varepsilon\left(Y, A^{\otimes s}\right)\right)$. Moreover, from definition (7) of $\delta_{\eta}(Y, A)$ it follows that

$$
\delta_{s \eta}\left(Y, A^{\otimes s}\right)=s^{k-2} \delta_{\eta}(Y, A), \text { for every } \eta \in(0, \varepsilon(Y, A)) \text { and } s \geq 1 .
$$

Example 1.4 In the special case $y=1$ (studied in [5]) the converse of (8) is also true, i.e.,

$$
\delta_{\eta}(Y, A)>0 \text { for some } \eta \in(0, \varepsilon(Y, A)) \Longrightarrow \delta(Y, A)>0 .
$$

Indeed in this case $y-k+t+2=-k+t+3$, whence $s_{-k+t+3}(N)=0$ for $t<k-3$. As $s_{1}(N)=-\operatorname{deg} N$,

$$
\delta(Y, A)=\varepsilon(Y, A)^{k-3}(\varepsilon(Y, A) \operatorname{deg} N-(k-2) d) \text { and } \delta_{\eta}(Y, A)=\eta^{k-3}(\eta \operatorname{deg} N-(k-2) d),
$$

where $d:=\int_{Y} c_{1}\left(A_{Y}\right)$ is the degree of $Y$ with respect to the polarization $A$. In particular we recover the definitions of $\delta(Y, A)$ and $\delta_{\eta}(Y, A)$ given in [28] (for $\left.k=3\right)$ and in [5] (for $k \geq 4$ ) when $Y$ is a curve. Moreover, in this case, we also get that $\delta_{\eta}(Y, A)>0$ implies $\operatorname{deg} N>0$, whence

$$
\delta(Y, A)=\varepsilon^{k-3}(\varepsilon \operatorname{deg} N-(k-2) d)>\eta^{k-3}(\eta \operatorname{deg} N-(k-2) d)=\delta_{\eta}(Y, A)>0,
$$

since $\varepsilon:=\varepsilon(Y, A)>\eta$.

Example 1.5 If $Y$ is a surface formulae (6), (7) become

$$
\begin{gathered}
\delta(Y, A)=-\varepsilon^{k-4}\left(\varepsilon^{2} \int_{Y} s_{2}(N)-\varepsilon(k-2) \int_{Y} s_{1}(N) \cdot c_{1}\left(A_{Y}\right)-\frac{(k-2)(k-3)}{2} \int_{Y} c_{1}\left(A_{Y}\right)^{\cdot 2}\right), \\
\delta_{\eta}(Y, A)=-\eta^{k-4}\left(\eta^{2} \int_{Y} s_{2}(N)-\eta(k-2) \int_{Y} s_{1}(N) \cdot c_{1}\left(A_{Y}\right)-\frac{(k-2)(k-3)}{2} \int_{Y} c_{1}\left(A_{Y}\right)^{\cdot 2}\right),
\end{gathered}
$$

where $\varepsilon:=\varepsilon(Y, A)$ and $\eta \in(0, \varepsilon)$. From the equalities $s_{1}(N)=-c_{1}(N)$ and $s_{2}(N)=c_{1}(N)^{\cdot 2}-$ $c_{2}(N)$ (which follow from the definition of Segre and Chern classes, see Example 1.12 below) we get

$$
\delta=-\varepsilon^{k-4}\left(\varepsilon^{2} \int_{Y}\left(c_{1}(N)^{\cdot 2}-c_{2}(N)\right)-\varepsilon(k-2) \int_{Y} c_{1}(N) \cdot c_{1}\left(A_{Y}\right)+\frac{(k-2)(k-3)}{2} \operatorname{deg}(Y)\right),
$$

and

$$
\delta_{\eta}=-\eta^{k-4}\left(\eta^{2} \int_{Y}\left(c_{1}(N)^{\cdot 2}-c_{2}(N)\right)-\eta(k-2) \int_{Y} c_{1}(N) \cdot c_{1}\left(A_{Y}\right)+\frac{(k-2)(k-3)}{2} \operatorname{deg}(Y)\right),
$$

where $\delta:=\delta(Y, A), \delta_{\eta}:=\delta_{\eta}(Y, A)$, and $\operatorname{deg}(Y)=\int_{Y} c_{1}\left(A_{Y}\right)^{\cdot 2}$ is the degree of $Y$ with respect to the polarization $A$.

Here are a couple of examples regarding the computation of Seshadri constants.

Example 1.6 (Complete intersections) Let $X$ be a submanifold of dimension $k$ of the projective space $\mathbb{P}^{n}$, and let $Y$ be a submanifold of dimension $y$ of $X$. Assume that $Y$ is a (scheme theoretic) complete intersection in $X$, i.e., $Y$ is the transversal intersection $Y=F_{1} \cap \cdots \cap F_{k-y}$ of $k-y$ hypersurfaces $F_{i} \subset X, i=1, \ldots, k-y$, of $\mathbb{P}^{n}$. Let $A=\mathcal{O}_{X}(1)$. Let $d_{i}=\operatorname{deg} F_{i}$, $i=1, \ldots, k-y$, and assume $d_{1} \geq \cdots \geq d_{k-y}$. Then the Seshadri constant of $(Y, A)$ is

$$
\varepsilon(Y, A)=\frac{1}{d_{1}} .
$$


It is a general well known fact that $d_{1} A^{*}-E$ is spanned by its global sections, which implies that $\varepsilon(Y, A) \geq \frac{1}{d_{1}}$ (compare with Example 1.7 below).

Therefore, to prove the equality, it is enough to show that $d_{1} A^{*}-E$ is not ample. To see this note that the restriction of $d_{1} A^{*}-E$ to the section of $E \rightarrow Y$ corresponding to the quotient $N^{\vee} \rightarrow\left(-d_{1} A^{*}\right) \rightarrow 0$ is the trivial bundle (see also [9, Corollary 2.5]).

Example 1.7 (Varieties defined in a given degree) Notation as in Definition [1.2. Let $\mathcal{J}_{Y}$ be the ideal sheaf of $Y$ in $X$. We say that $Y$ is defined by $A$ in degree $t$ if either $\mathcal{J}_{Y}(t):=A^{\otimes t} \otimes \mathcal{J}_{Y}$ is generated by its global sections, or, equivalently, if $Y$ is the scheme theoretic intersection of all $\operatorname{divisors}_{\operatorname{div}_{X}}(s)$, with $s \in H^{0}\left(X, A^{\otimes t} \otimes \mathcal{J}_{Y}\right) \backslash\{0\}$. In this case, from [5. Lemma 1.5 and Corollary 1.6], we know that

$$
\varepsilon(Y, A) \geq \frac{1}{t}
$$

The degree $t=2$ case with $X=\mathbb{P}^{k}, Y$ a submanifold of $\mathbb{P}^{k}$ (not a linear subspace of $\mathbb{P}^{k}$ ) and $A=\mathcal{O}_{\mathbb{P} k}(1)$ is of special interest in the sequel. Take a line $\ell$ meeting $Y$ in at least two points but not contained in $Y$. Note that such a line exists, since otherwise $Y$ would be a linear subspace in $\mathbb{P}^{k}$. Let $\ell^{\prime}$ be the proper transform of $\ell$ under the blowing up $\pi$ of $\mathbb{P}^{k}$ along $Y$. Then

$$
\pi^{*} \mathcal{J}_{Y}(2) \cdot \ell^{\prime}=\left(2 A^{*}-E\right) \cdot \ell^{\prime}=2-E \cdot \ell^{\prime} \leq 0 .
$$

Therefore $2 A^{*}-E$ is spanned but not ample. It thus follows that

$$
\varepsilon(Y, A)=\frac{1}{2} \text {. }
$$

Many of the varieties $Y \subset \mathbb{P}^{k}$ we will deal with throughout the paper-varieties of minimal degree, Segre varieties - are indeed generated in degree two, so that they satisfy condition (13). Determinantal varieties give further examples of varieties defined in a given degree (see [9, §2]).

Remark 1.8 As $(0, \varepsilon(Y, A) \cap \mathbb{Q})$ is dense in $(0, \varepsilon(Y, A))$, it follows that there exists $\eta \in$ $(0, \varepsilon(Y, A))$ such that $\delta_{\eta}(Y, A)>0$ if and only if there exists $\eta \in(0, \varepsilon(Y, A)) \cap \mathbb{Q}$ such that $\delta_{\eta}(Y, A)>0$.

For the simplicity of notation, when there is no danger of confusion, we shall make no difference between the line bundle $A^{*}$ and the Chern class $c_{1}\left(A^{*}\right)$. Choose now $\eta \in(0, \varepsilon(Y, A)) \cap$ $\mathbb{Q}$ such that the $\mathbb{Q}$-line bundle $A^{*} \otimes \mathcal{O}_{X_{Y}}(-\eta E)=\mathcal{O}_{X_{Y}}\left(A^{*}-\eta E\right)$ is ample. Write $\eta=\frac{n}{m}$, with $m$ and $n$ positive integers. Then the divisor $m A^{*}-n E$ is ample by the choice of $\eta=\frac{n}{m}$. Assume that in the ample linear system $\left|m A^{*}-n E\right|$ there exist divisors $H_{1}, \ldots, H_{k-2}$ such that $\mathcal{H}$ is a smooth surface and $Y^{\prime}$ is a smooth curve, where

$$
Y^{\prime}:=E \cap \mathcal{H} \text {, and } \mathcal{H}:=H_{1} \cap \cdots \cap H_{k-2} .
$$

Note that this condition can be easily realized because if we multiply $m, n$ by a suitable positive large integer $\lambda$ (which does not change $\eta$ ), the linear system $\left|\lambda m A^{*}-\lambda n E\right|$ becomes very ample, and then apply Bertini's theorem.

Lemma 1.9 Let $\eta=\frac{n}{m} \in(0, \varepsilon(Y, A)) \cap \mathbb{Q}$, with $n$ and $m$ positive integers. Then $\delta_{\eta}(Y, A)>0$ if and only if $\left(Y^{\prime \cdot 2}\right)_{\mathcal{H}}>0$, where $Y^{\prime}$ and $\mathcal{H}$ are defined by (14) and $\left(Y^{\prime \cdot 2}\right)_{\mathcal{H}}:=\int_{\mathcal{H}} Y^{\prime \cdot 2}$.

Proof. As $\left(Y^{\prime \cdot 2}\right)_{\mathcal{H}}=\int_{\mathcal{H}} E_{\mathcal{H}}^{\cdot 2}=\int_{X_{Y}} E^{\cdot 2} \cdot\left(m c_{1}\left(A^{*}\right)-n E\right)^{\cdot(k-2)}$, by Lemma 1.1 we get

$$
\begin{aligned}
\frac{1}{m^{k-2}}\left(Y^{\prime \cdot 2}\right)_{\mathcal{H}} & =\int_{X_{Y}} E^{\cdot 2} \cdot\left(c_{1}\left(A^{*}\right)-\eta E\right)^{\cdot(k-2)} \\
& =\sum_{t=0}^{k-2}(-1)^{t} \eta^{t}\left(\begin{array}{c}
k-2 \\
t
\end{array}\right) \int_{X_{Y}} E^{\cdot(t+2)} \cdot c_{1}\left(A^{*}\right)^{\cdot(k-2-t)}
\end{aligned}
$$




$$
\begin{aligned}
& =\sum_{t=0}^{k-2}(-1)^{t}\left(\begin{array}{c}
k-2 \\
t
\end{array}\right) \eta^{t}(-1)^{t+1} \int_{Y} s_{y-k+t+2}(N) \cdot c_{1}\left(A_{Y}\right)^{\cdot(k-t-2)} \\
& =-\sum_{t=0}^{k-2}\left(\begin{array}{c}
k-2 \\
t
\end{array}\right) \eta^{t} \int_{Y} s_{y-k+t+2}(N) \cdot c_{1}\left(A_{Y}\right)^{\cdot(k-t-2)} \\
& =\delta_{\eta}(Y, A)
\end{aligned}
$$

Now we come up to the following basic general definition.

Definition 1.10 Under the above notation, we say that the submanifold $Y$ of $X$ is Seshadri $A$-big (or big with respect to a fixed polarization $A$ of $X$ ) if there exists an $\eta \in(0, \varepsilon(Y, A))$ such that $\delta_{\eta}(Y, A)>0$. By Remark 1.8 the latter condition is also equivalent to the existence of an $\eta^{\prime} \in(0, \varepsilon(Y, A)) \cap \mathbb{Q}$ such that $\delta_{\eta^{\prime}}(Y, A)>0$. Or more geometrically, in view of Lemma 1.10, the submanifold $Y$ of $X$ is Seshadri $A$-big if and only if there exists $\eta=\frac{n}{m} \in(0, \varepsilon(Y, A)) \cap \mathbb{Q}$ such that $\left(Y^{\prime \cdot 2}\right)_{\mathcal{H}}>0$. As $Y^{\prime}$ is a smooth irreducible curve on the smooth projective surface $\mathcal{H}$, the fact that $Y \subset X$ is Seshadri $A$-big amounts to the fact that the normal bundle $N_{Y^{\prime} \mid \mathcal{H}}=$ $\mathcal{O}_{\mathcal{H}}\left(Y^{\prime}\right) \otimes \mathcal{O}_{Y^{\prime}}$ of $Y^{\prime}$ in $\mathcal{H}$ is ample on $Y^{\prime}$.

We also say that $Y \subset X$ is Seshadri A-ample (or ample with respect to the polarization $A$ ) if $Y^{\prime}$ is an ample divisor on $\mathcal{H}$. Clearly, if $Y$ is Seshadri $A$-ample then $Y$ is Seshadri $A$-big. The Seshadri $A$-ampleness is also an open condition, i.e., if it is satisfied for some $\eta=\frac{n}{m} \in(0, \varepsilon(Y, A)) \cap \mathbb{Q}$, then it also satisfied for every $\eta^{\prime} \in(\eta-\nu, \eta+\nu) \cap \mathbb{Q}$, with $\nu>0$ sufficiently small.

Remark 1.11 From Definition 1.10 and (9) it follows immediately that a submanifold $Y$ of $X$ is Seshadri $A$-big if and only if it is Seshadri $A^{\otimes s}$-big for each integer $s \geq 1$. Using this and Theorem 5.1 below we shall see that a submanifold $Y$ of $X$ is Seshadri $A$-ample if and only if it is Seshadri $A^{\otimes s}$-ample.

1.12 Some generalities on Segre and Chern classes. It is usually more convenient to express the numerical invariants $\delta(Y, A)$ and $\delta_{\eta}(Y, A)$ in terms of the Chern classes of the normal bundle $N$ of a closed smooth $y$-dimensional subvariety $Y$ of the projective smooth $k$-dimensional variety $X$. This is due to the fact that in general a vector bundle $\mathcal{E}$ of rank $r$ on an algebraic variety $Z$ has Chern classes $c_{i}(\mathcal{E})=0$ for every $i>\min \{\operatorname{dim}(Z), r\}$. For this we recall the identity $s_{t}(\mathcal{E}) \cdot c_{t}(\mathcal{E})=1$ between the Segre and the Chern polynomials $s_{t}(\mathcal{E})$ and $c_{t}(\mathcal{E})$, where

$$
s_{t}(\mathcal{E})=\sum_{i=0}^{y} s_{i}(\mathcal{E}) t^{i} \text { and } c_{t}(\mathcal{E})=\sum_{i=0}^{y} c_{i}(\mathcal{E}) t^{i}, \text { with } s_{0}(\mathcal{E})=c_{0}(\mathcal{E})=1,
$$

which holds for every vector bundle $\mathcal{E}$. (See [12, p. 50] for the definition of the Chern polynomials.) And, for every $n \geq 1$, we have the general recurrence formula

$$
s_{n}(\mathcal{E})=-s_{n-1}(\mathcal{E}) \cdot c_{1}(\mathcal{E})-s_{n-2}(\mathcal{E}) \cdot c_{2}(\mathcal{E})-\cdots-s_{1}(\mathcal{E}) \cdot c_{n-1}(\mathcal{E})-c_{n}(\mathcal{E}) .
$$

In particular,

$$
\begin{gathered}
s_{1}(\mathcal{E})=-c_{1}(\mathcal{E}), \quad s_{2}(\mathcal{E})=c_{1}(\mathcal{E})^{\cdot 2}-c_{2}(\mathcal{E}), \quad s_{3}(\mathcal{E})=2 c_{1}(\mathcal{E}) \cdot c_{2}(\mathcal{E})-c_{1}(\mathcal{E})^{\cdot 3}-c_{3}(\mathcal{E}), \\
s_{4}(\mathcal{E})=-3 c_{1}(\mathcal{E})^{\cdot 2} \cdot c_{2}(\mathcal{E})+c_{1}(\mathcal{E})^{\cdot 4}+c_{2}(\mathcal{E})^{\cdot 2}+2 c_{1}(\mathcal{E}) \cdot c_{3}(\mathcal{E})-c_{4}(\mathcal{E}), \ldots
\end{gathered}
$$

Recall also that $c_{1}(\varepsilon)=c_{1}(\operatorname{det}(\varepsilon))$, where $\operatorname{det}(\varepsilon)=\wedge^{r} \mathcal{E}$, see [12, p. 55]. 
Take now $Z=Y$ and $\mathcal{E}=N$ (the normal bundle of $Y$ in $X$ ). If for example $\operatorname{codim}_{X}(Y)=2$ and $k \leq 6$ we have $c_{i}(N)=0$ for every $i \geq 3$, whence the above formulae and (7) yield for $\delta_{\eta}(Y, A)$ the following expressions:

$$
\begin{aligned}
\delta_{\eta}(Y, A)= & -\eta^{2} \int_{Y}\left(c_{1}(N)^{\cdot 2}-c_{2}(N)\right) \\
& +2 \eta \int_{Y} c_{1}(N) \cdot c_{1}\left(A_{Y}\right)-\operatorname{deg}(Y), \text { for }(k, y)=(4,2) ; \\
\delta_{\eta}(Y, A)= & \left.-\eta^{3} \int_{Y}\left(2 c_{1}(N) \cdot c_{2}(N)\right)-c_{1}(N)^{\cdot 3}\right) \\
& -3 \eta^{2} \int_{Y}\left(c_{1}(N)^{\cdot 2}-c_{2}(N)\right) \cdot c_{1}\left(A_{Y}\right) \\
& +3 \eta \int_{Y} c_{1}(N) \cdot c_{1}\left(A_{Y}\right)^{\cdot 2}-\operatorname{deg}(Y), \text { for }(k, y)=(5,3) ; \\
\delta_{\eta}(Y, A)= & \eta^{4} \int_{Y}\left(3 c_{1}(N)^{\cdot 2} \cdot c_{2}(N)-c_{2}(N)^{\cdot 2}-c_{1}(N)^{\cdot 4}\right) \\
& -4 \eta^{3} \int_{Y}\left(2 c_{1}(N) \cdot c_{2}(N)-c_{1}(N)^{\cdot 3}\right) \cdot c_{1}\left(A_{Y}\right) \\
& -6 \eta^{2} \int_{Y}\left(c_{1}(N)^{\cdot 2}-c_{2}(N)\right) \cdot c_{1}\left(A_{Y}\right)^{\cdot 2} \\
+ & 4 \eta \int_{Y} c_{1}(N) \cdot c_{1}\left(A_{Y}\right)^{\cdot 3}-\operatorname{deg}(Y), \text { for }(k, y)=(6,4) .
\end{aligned}
$$

\section{The one-codimensional case}

Let us start with the simplest case when $\operatorname{codim}_{X}(Y)=1$, i.e., $y=k-1$. In this case, under the notation of diagram (3), we have $X_{Y}=X$ and $E=Y$. Therefore

$$
\varepsilon(Y, A)=\sup \{\eta \in \mathbb{Q} \mid A-\eta Y \text { is ample }\} .
$$

The following result already provides many examples of Seshadri $A$-big submanifolds of codimension one.

Proposition 2.1 Let $(X, A)$ be a polarized manifold of dimension $k \geq 2$, and let $Y$ be a submanifold of $X$ of dimension $y=k-1$. If the normal bundle $N=\mathcal{O}_{X}(Y) \otimes \mathcal{O}_{Y}$ of $Y$ in $X$ is ample then $Y$ is Seshadri A-big. If $k=2$ the converse also holds.

Proof. Since $y=k-1$, formula (7) yields

$$
\begin{aligned}
\delta_{\eta}(Y, A) & =-\sum_{t=0}^{k-2}\left(\begin{array}{c}
k-2 \\
t
\end{array}\right) \eta^{t} \int_{Y} s_{t+1}(N) \cdot c_{1}\left(A_{Y}\right)^{\cdot(k-t-2)} \\
& =-\sum_{t=0}^{k-2}\left(\begin{array}{c}
k-2 \\
t
\end{array}\right) \eta^{t}(-1)^{t+1} \int_{Y} c_{1}(N)^{\cdot(t+1)} \cdot c_{1}\left(A_{Y}\right)^{\cdot(k-t-2)} \\
& =\int_{Y} c_{1}(N) \cdot c_{1}\left(A_{Y}-\eta N\right)^{\cdot(k-2)}
\end{aligned}
$$


Thus $\delta_{\eta}(Y, A)>0$ because $N$ and $A_{Y}-\eta N$ are ample on $Y$ for every $\eta \in(0, \varepsilon(Y, A))$ (the latter as the restriction of the ample line bundle $A-\eta Y$, via (20) $)$. Therefore $Y$ is Seshadri A-big.

Conversely, assume $k=2$ and $\delta_{\eta}(Y, A)>0$. Then $\operatorname{deg}(N)=\int_{Y} c_{1}(N)=\delta_{\eta}(Y, A)>0$, whence $N$ is ample because it is a line bundle on the curve $Y$.

In order to provide more examples of Seshadri $A$-big submanifolds in codimension one we need the following straightforward consequence of Lemma 1.9 and Definition 1.10 .

Lemma 2.2 Let $(X, A)$ be a polarized manifold of dimension $k \geq 2$, and let $Y$ be a submanifold of $X$ of dimension $y=k-1$. Then the following conditions are equivalent:

1. $Y$ is Seshadri A-big.

2. There exists an $\eta=\frac{n}{m} \in(0, \varepsilon(Y, A)) \cap \mathbb{Q}$, with $m$ and $n$ positive integers, such that the line bundle $m A-n Y$ is very ample on $X$ and for every general divisors $H_{1}, \ldots, H_{k-2} \in$ $|m A-n Y|$ one has $\left(Y^{\prime \cdot 2}\right)_{\mathcal{H}}:=\int_{\mathcal{H}} Y^{\prime \cdot 2}>0$, where $Y^{\prime}:=Y \cap H_{1} \cap \cdots \cap H_{k-2}$ and $\mathcal{H}:=H_{1} \cap \cdots \cap H_{k-2}($ see (14) $)$.

3. There exist an $\eta=\frac{n}{m} \in(0, \varepsilon(Y, A)) \cap \mathbb{Q}$, with $m$ and $n$ positive integers, and divisors $H_{1}, \ldots, H_{k-2} \in|m A-n Y|$ such that $\mathcal{H}:=H_{1} \cap \cdots \cap H_{k-2}$ is a smooth surface, $Y^{\prime}:=$ $Y \cap H_{1} \cap \cdots \cap H_{k-2}$ a smooth curve, and $\left(Y^{\prime 2}\right)_{\mathcal{H}}:=\int_{\mathcal{H}} Y^{\prime \cdot 2}>0$.

Corollary 2.3 Let $(X, A)$ be a polarized manifold of dimension $k \geq 3$, and let $Y$ be a submanifold of $X$ of dimension $y=k-1$. Assume that the normal bundle $N=\mathcal{O}_{X}(Y) \otimes \mathcal{O}_{Y}$ of $Y$ in $X$ satisfies the following condition:

$$
\left.\operatorname{deg}\left(N \otimes \mathcal{O}_{C}\right)\right)>0 \text { for every smooth curve } C \text { in } Y \text { with normal bundle } N_{C \mid Y} \text { ample. }
$$

Then $Y$ is Seshadri A-big.

Proof. It is enough to check condition 2) of Lemma 2.2 Recall that for $\eta=\frac{n}{m} \in(0, \varepsilon(Y, A)) \cap \mathbb{Q}$, with $m$ and $n$ positive integers such that the line bundle $m A-n Y$ is very ample on $X$, and for every general divisors $H_{1}, \ldots, H_{k-2} \in|m A-n Y|$, the intersection $Y^{\prime}=Y \cap \mathcal{H}$ is proper, where $\mathcal{H}=H_{1} \cap \cdots \cap H_{k-2}$ is a smooth surface. Therefore $N_{Y^{\prime} \mid \mathcal{H}} \cong N \otimes \mathcal{O}_{Y^{\prime}}$, and hence $N_{Y^{\prime} \mid \mathcal{H}}$ is ample. It follows that $\left(Y^{\prime \cdot 2}\right)_{\mathcal{H}}=\operatorname{deg}\left(N_{Y^{\prime} \mid \mathcal{H}}\right)=\operatorname{deg}\left(N \otimes \mathcal{O}_{Y^{\prime}}\right)>0$ by hypothesis (21).

Examples 2.4 Corollary 2.3 leads to explicit construction of $A$-big divisors.

i) In characteristic zero there are examples of line bundles $N$ on some smooth projective varieties $Y$ of dimension $\geq 2$ such that $\operatorname{deg}\left(N \otimes \mathcal{O}_{C}\right)>0$ for every irreducible curve $C$ on $Y$, but which are not ample. The first of such examples has been found by Mumford (see e.g. 22, Example 10.6, p. 56]). Specifically, let $B$ be a smooth projective curve of genus $g \geq 2$ over $\mathbb{C}$ and let $E$ be a vector bundle of rank 2 and degree 0 over $B$ such that all symmetric powers $\mathbf{S}^{m}(E), m \geq 1$, are stable (one can show that such vector bundles $E$ do exist). Let $Y=\mathbb{P}(E)$ be the projective bundle associated to $E$ and set $N=\mathcal{O}_{\mathbb{P}(E)}(1)$. Then $\int_{Y} c_{1}(N)^{\cdot 2}=\operatorname{deg}(E)=0$ (in particular $N$ is not ample), but one can show that condition (21) of Corollary 2.3 is satisfied [22, Example 10.6, p. 56]. Moreover in this case one has $H^{0}(Y, N)=0$.

Starting with Mumford's example $Y=\mathbb{P}(E)$ above, we may easily construct an example of a polarized threefold $(X, A)$ containing $Y$ as a divisor with normal bundle $N_{Y \mid X}=N=$ $\mathcal{O}_{\mathbb{P}(E)}(1)$ not ample, but satisfying condition (21) of Corollary 2.3. Indeed, let $X^{\prime}:=\mathbb{V}\left(N^{-1}\right)=$ $\operatorname{Spec}\left(\mathbf{S}\left(N^{-1}\right)\right)$ be the vector bundle over $Y$ associated to the dual of $N$ (with $\mathbf{S}\left(N^{-1}\right)=$ $\overline{\oplus_{m=0}^{\infty}} N^{\otimes-m}$ ) and let $i: Y \hookrightarrow X^{\prime}$ be the zero section of $\mathbb{V}\left(N^{-1}\right)$ (which corresponds to the canonical surjection $\left.\oplus_{m=0}^{\infty} N^{\otimes-m} \rightarrow \mathcal{O}_{Y}\right)$. Then the normal bundle of $i(Y)$ in $X^{\prime}$ is isomorphic 
to $N$. Since $X^{\prime}$ is smooth and only quasi-projective, take the natural projective closure $X:=$ $\mathbb{P}\left(N^{-1} \oplus \mathcal{O}_{Y}\right)$ of $\left.X^{\prime}:=\mathbb{V}\left(N^{-1}\right)\right)$. Finally, choosing any polarization $A$ on $X$ we get an example of a polarized threefold $(X, A)$ containing $i(Y) \cong Y$ with normal bundle (isomorphic to) $N$ which is not ample, but it satisfies the hypotheses of Corollary 2.3. Then $i(Y)$ is Seshadri $A$ big in $X$.

ii) Note also that there is an example (due to C.P. Ramanujam [22, Example 10.8, p. 57]) of a projective threefold $Y$ over $\mathbb{C}$ and a line bundle $N$ on $Y$ which is not ample, but still satisfies condition (21) of Corollary 2.3. Moreover, in Ramanujam's example one has $\int_{Y} c_{1}(N)^{\cdot 3}>0$ and $H^{0}(Y, N) \neq 0$. Then, exactly as in case ii) above, starting with the threefold $Y$ and with the line bundle $N$, one can construct an example of a polarized fourfold $(X, A)$ containing $Y$ as a divisor with normal bundle $N$ and satisfying the hypotheses of Corollary 2.3 (taking $X:=\mathbb{P}\left(N^{-1} \oplus \mathcal{O}_{Y}\right)$ and for $A$ any polarization on $\left.X\right)$. Therefore $Y$ is Seshadri $A$-big in $X$.

iii) Another interesting application of Corollary 2.3 starts with the following example due to Serre (see [22, p. 232, Example 3.2]). Let $B$ be an elliptic curve over $\mathbb{C}$, and consider a non-trivial extension of vector bundles

$$
0 \longrightarrow \mathcal{O}_{B} \longrightarrow E \stackrel{\beta}{\longrightarrow} \mathcal{O}_{B} \longrightarrow 0 .
$$

Indeed, the isomorphism classes of such extensions are classified by $H^{1}\left(B, \mathcal{O}_{B}\right)$, which is a one-dimensional vector space because $B$ is an elliptic curve. Thus non-trivial extensions of type (22) exist. Consider the geometrically ruled surface $Y:=\mathbb{P}(E)$ associated to the rank two vector bundle $E$, and denote by $p: Y \rightarrow B$ the canonical projection. Let $C$ be the section of $p$ corresponding to the surjection $\beta$. Since the extension (22) is non-trivial one can show (22, p. 232, Example 3.2]) that the following properties hold.

a) $\int_{Y} C^{\cdot 2}=0$ and, in particular, $Y \backslash C$ is not an affine open subset of $Y$.

b) The associated analytic space $(X \backslash Y)^{\text {an }}$ is biholomorphically isomorphic to $\mathbb{C}^{*} \times \mathbb{C}^{*}$, and therefore is a Stein manifold.

Set $N:=\mathcal{O}_{Y}(C)$ and $V:=Y \backslash C$. From a) and b) it follows that any irreducible curve $C^{\prime}$ of $Y, C^{\prime} \neq C$, satisfies the following properties: $C^{\prime} \cap V \neq \varnothing$ and $C^{\prime} \nsubseteq V$. Such properties imply that for every irreducible curve $C^{\prime} \neq C$ on $Y$ one has $\int_{Y} c_{1}(N) \cdot c_{1}\left(\mathcal{O}_{Y}\left(C^{\prime}\right)\right)>0$, i.e., $\operatorname{deg}\left(N \otimes \mathcal{O}_{C^{\prime}}\right)>0$ for every irreducible curve $C^{\prime}$ different from $C$. Recalling also that the normal bundle of $C$ in $Y$ has degree 0, we then proved that hypothesis (21) of Corollary 2.3 is satisfied. At this point, setting $X=\mathbb{P}\left(N^{-1} \oplus \mathcal{O}_{Y}\right)$ and taking $A$ any polarization on $X$, we get a polarized threefold $(X, A)$ containing a divisor $Y$ (the zero section of the bundle) with normal bundle $N=\mathcal{O}_{Y}(C)$. Then by Corollary 2.3 we conclude that $Y$ is Seshadri $A$-big in $X$.

Remark 2.5 Further examples of non-ample line bundles $N$ on a projective manifold $Y$ such that $\operatorname{deg}\left(N \otimes \mathcal{O}_{C}\right)>0$ for every irreducible curve $C$ in $Y$ can be found in $[8$. Examples 3.13 and 3.14]. Starting with such a pair $(Y, N)$ and performing the construction $X=\mathbb{P}\left(N^{-1} \oplus \mathcal{O}_{Y}\right)$ as in Examples 2.4 we get an effective divisor $Y$ in $X$ which is Seshadri $A$-big with respect to any polarization $A$ of $X$. Moreover, in these examples (including Example 2.4 iii), in which $N$ is not nup in the sense of [8] the projective manifold $X=\mathbb{P}\left(N^{-1} \oplus \mathcal{O}_{Y}\right)$ contains $Y$ as the "zero section" such that $Y$ is a Seshadri $A$-big divisor in $X$ with respect to any polarization $A$ on $X$. Then the "section at infinity" (the image $Z$ of the closed immersion $Y \subset X=\mathbb{P}\left(N^{-1} \oplus \mathcal{O}_{Y}\right)$ corresponding to the canonical surjection $N^{-1} \oplus \mathcal{O}_{Y} \rightarrow \mathcal{O}_{Y}$ ) is a hypersurface of $X$ such that $Y \cap Z=\varnothing$. However, by Theorem 5.1 below, it will follow that in all these examples $Y$ cannot be Seshadri $A$-ample in $X$. 


\section{Seshadri $A$-big submanifolds of higher codimension}

In this section we give a series of relevant examples of submanifolds $Y$ of dimension $\geq 2$ and of codimension $\geq 2$ in a polarized manifold $(X, A)$ that are Seshadri $A$-big, giving a good motivation for the study of this notion in general. Examples of Seshadri $A$-big curves in a polarized $k$-fold can be found in 28 , if $k=3$, and subsequently in [5], if $k \geq 4$. Proposition 3.16 at the end of this section will show that Seshadri $A$-bigness is stable under finite coverings.

3.1 Complete intersections in codimension 2. Assume that $Y$ is the complete intersection surface in a fourfold $X$, embedded in $\mathbb{P}^{n}$, with two hypersurfaces of $\mathbb{P}^{n}$ of degrees $d_{1} \geq d_{2}$. Let $A=\mathcal{O}_{X}(1)$. By Example 1.6, we have $\varepsilon=\varepsilon(Y, A)=\frac{1}{d_{1}}$. Moreover, $d:=\int_{Y} c_{1}\left(\mathcal{O}_{Y}(1)\right)^{-2}$ is the degree of $Y$. Then equality (17) immediately gives

$$
\delta(Y, A)=d\left(-\varepsilon^{2}\left(d_{1}^{2}+d_{2}^{2}+d_{1} d_{2}\right)+2 \varepsilon\left(d_{1}+d_{2}\right)-1\right)=d\left(\frac{d_{2}}{d_{1}}-\frac{d_{2}^{2}}{d_{1}^{2}}\right) .
$$

Therefore $\delta(Y, A)>0$ if $d_{1}>d_{2}$, and $\delta(Y, A)=0$ if $d_{1}=d_{2}$. In the former case we get $\delta_{\eta}(Y, A)>0$, and in the latter case we have

$$
\delta_{\eta}(Y, A)=d\left(-3 d_{1}^{2} \eta^{2}+4 d_{1} \eta-1\right) .
$$

Thus the polynomial $f(x)=-3 d_{1}^{2} x^{2}+4 d_{1} x-1$ (with real coefficients), which has the roots $\frac{1}{d_{1}}$ and $\frac{1}{3 d_{1}}$, assumes positive values for every $x \in\left(\frac{1}{3 d_{1}}, \frac{1}{d_{1}}\right)$. Then, for every $d_{1} \geq d_{2}$, we have $\delta_{\eta}(Y, A)>0$ for every $\eta \in\left(\frac{1}{3 d_{1}}, \frac{1}{d_{1}}\right)$.

Assume now that $Y$ is the complete intersection threefold of the fivefold $X$, embedded in $\mathbb{P}^{n}$, with two hypersurfaces of $\mathbb{P}^{n}$ of degrees $d_{1} \geq d_{2}$. Let $A=\mathcal{O}_{X}(1)$. By Example 1.6 again, we have $\varepsilon(Y, A)=\frac{1}{d_{1}}$. Let $d:=\int_{Y} c_{1}\left(\mathcal{O}_{Y}(1)\right)^{\cdot 3}$ be the degree of $Y$. Proceeding similarly as above and using (18) we immediately get:

$$
\delta(Y, A)=d\left(\left(\frac{d_{2}}{d_{1}}\right)^{3}-2\left(\frac{d_{2}}{d_{1}}\right)^{2}+\frac{d_{2}}{d_{1}}\right)=\frac{d d_{2}}{d_{1}}\left(\frac{d_{2}}{d_{1}}-1\right)^{2} .
$$

It follows that $\delta(Y, A) \geq 0$, with equality if and only if $d_{1}=d_{2}$. On the other hand,

$$
\delta_{\eta}(Y, A)=d\left(-1+3\left(d_{1}+d_{2}\right) \eta-3\left(d_{1}^{2}+d_{1} d_{2}+d_{2}^{2}\right) \eta^{2}+\left(d_{1}^{3}+d_{1}^{2} d_{2}+d_{1} d_{2}^{2}+d_{2}^{3}\right) \eta^{3}\right) .
$$

Then if in the polynomial (with real coefficients)

$$
\left(d_{1}^{3}+d_{1}^{2} d_{2}+d_{1} d_{2}^{2}+d_{2}^{3}\right) x^{3}-3\left(d_{1}^{2}+d_{1} d_{2}+d_{2}^{2}\right) x^{2}+3\left(d_{1}+d_{2}\right) x-1
$$

we put $d_{1}=d_{2}$, we get the polynomial

$$
P(x)=4 d_{1}^{3}-9 d_{1}^{2} x^{2}+6 d_{1} x-1=4 d_{1}^{3}\left(x-\frac{1}{d_{1}}\right)^{2}\left(x-\frac{1}{4 d_{1}}\right) .
$$

As above, it follows that $\delta_{\eta}(Y, A)>0$ for every $\eta \in\left(\frac{1}{4 d_{1}}, \frac{1}{d_{1}}\right)$.

Recalling Lemma 1.9, the previous computations leads to the following result: if $Y$ is a smooth complete intersection of codimension 2 of a projective submanifold $X$ of dimension $k=4,5$ of $\mathbb{P}^{n}$, then $Y$ is Seshadri $\mathcal{O}_{X}(1)$-big. However, from a more conceptual point of view, we shall generalize this result to all complete intersections of codimension 2, see Proposition 3.4 below.

Notice that in codimension $k-y \geq 3$ not every complete intersection is Seshadri $\mathcal{O}_{X}(1)$-big. This already happens for curves. Precisely, if $Y$ is a smooth complete intersection curve in $X$ 
( $X \subseteq \mathbb{P}^{n}$ ) of multidegree $d_{1} \geq d_{2} \geq \cdots \geq d_{k-1} \geq 1$, then $Y$ is Seshadri $\mathcal{O}_{X}(1)$-big if and only if $d_{2}+\cdots+d_{k-1}>(k-3) d_{1}$, see [5, Example 1.7].

Assume now that $(k, y)=(5,2)$, i.e., $Y$ is a smooth complete intersection surface of the 5 -fold $X \subset \mathbb{P}^{5}$ with three hypersurfaces $\mathbb{P}^{5}$ of degrees $d_{1} \geq d_{2} \geq d_{3} \geq 1$. Then the Seshadri constant is $\varepsilon\left(Y, \mathbb{P}^{5}\right)=\frac{1}{d_{1}}$ and by (12), for every $\eta \in\left(0, \frac{1}{d_{1}}\right) \cap \mathbb{Q}$, we have

$$
\delta_{\eta}\left(Y, \mathcal{O}_{X}(1)\right)=d \eta\left(-\left(d_{1}^{2}+d_{2}^{2}+d_{3}^{2}+d_{1} d_{2}+d_{1} d_{3}+d_{2} d_{3}\right) \eta^{2}+3\left(d_{1}+d_{2}+d_{3}\right) \eta-3\right) .
$$

Consider the polynomial function $f: \mathbb{R} \rightarrow \mathbb{R}$ defined by

$$
f(x)=-\left(d_{1}^{2}+d_{2}^{2}+d_{3}^{2}+d_{1} d_{2}+d_{1} d_{3}+d_{2} d_{3}\right) x^{2}+3\left(d_{1}+d_{2}+d_{3}\right) x-3 .
$$

For example, if we take $X=\mathbb{P}^{5}, d_{1}=3$ and $d_{2}=d_{3}=2$ we get $f(x)=-33 x^{2}+21 x-3$. Then $f\left(\frac{1}{3}\right)=\frac{1}{3}$, whence $Y$ is Seshadri $\mathcal{O}_{\mathbb{P}^{5}}(1)$-big.

If instead we take $X=\mathbb{P}^{5}, d_{1}=9$ and $d_{2}=d_{3}=2$ we get $f(x)=-129 x^{2}+39 x-3$, whose discriminant is $\Delta=-27<0$. We deduce that $f(x)<0$ for every $x \in \mathbb{R}$, which implies that in this case $Y$ is never Seshadri $\mathcal{O}_{\mathbb{P}^{5}}(1)$-big.

3.2 Zero loci of sections of ample vector bundles. Fix two integers $k$ and $y$ such that $1 \leq y \leq k-2$. Let $\mathcal{E}$ be an ample vector bundle of rank $k-y$ on a smooth projective $k$-dimensional variety $X, k \geq 3$, and set $A:=\operatorname{det}(\mathcal{E})$. As $\mathcal{E}$ is ample, a general result of Hartshorne [22 implies that $A$ is an ample line bundle on $X$. Let $s \in H^{0}(X, \mathcal{E})$ be a section such that its zero locus $Y:=Z(s)$ is a smooth $y$-dimensional subvariety of $X$. Then by a result of Sommese, $Y$ is connected (see [31, (1.16)], or also [15]). Then $s$ defines a map $s: \mathcal{O}_{X} \rightarrow \mathcal{E}$ and, taking the dual map $s^{*}: \mathcal{E}^{*} \rightarrow \mathcal{O}_{X}$, one gets the surjection $s^{*}: \mathcal{E}^{*} \rightarrow \mathcal{J}_{Y}$, where $\mathcal{J}_{Y}$ is the ideal sheaf of $Y$ in $X$. Thus we get a surjective map $\mathbf{S}\left(\mathcal{E}^{*}\right) \rightarrow \oplus_{i=0}^{\infty} \jmath_{Y}^{i}$, where $\mathbf{S}\left(\mathcal{E}^{*}\right)$ is the symmetric $\mathcal{O}_{X^{-}}$-algebra of $\mathcal{E}^{*}$. It follows that there is a closed immersion $i: X_{Y} \hookrightarrow \mathbb{P}\left(\mathcal{E}^{*}\right)$ of $X$-schemes such that $i^{*}\left(\mathcal{O}_{\mathbb{P}\left(\mathcal{E}^{*}\right)}(1)\right) \cong \mathcal{O}_{X_{Y}}(-E)$. Consider the canonical isomorphisms

$$
\wedge^{k-y-1} \mathcal{E} \cong \mathcal{E}^{*} \otimes \operatorname{det}(\mathcal{E})=\mathcal{E}^{*} \otimes A .
$$

It follows that there is a canonical isomorphism $\mathbb{P}\left(\wedge^{k-y-1} \mathcal{E}\right) \cong \mathbb{P}\left(\mathcal{E}^{*}\right)$ with the property that $\mathcal{O}_{\mathbb{P}\left(\wedge^{k-y-1} \mathcal{E}\right)}(1) \cong \mathcal{O}_{\mathbb{P}\left(\mathcal{E}^{*}\right)}(1) \otimes p^{*}(A)$, where $p: \mathbb{P}\left(\mathcal{E}^{*}\right) \rightarrow X$ is the canonical projection. As $\mathcal{E}$ is ample by hypothesis, a result of Hartshorne [20], or also [22], shows that $\wedge^{k-y-1} \mathcal{E}$ is also ample, i.e., $\mathcal{O}_{\mathbb{P}\left(\wedge^{k-y-1} \mathcal{E}\right)}(1) \cong \mathcal{O}_{\mathbb{P}\left(\mathcal{E}^{*}\right)}(1) \otimes p^{*}(A)$ is ample. Recalling the above immersion, this last fact implies that $A^{*}-E$ is ample on $X_{Y}$, whence $\varepsilon(Y, A)>1$.

Consider first the case when the vector bundle $\mathcal{E}$ is decomposable of rank 2 , i.e., $\mathcal{E}=L^{\prime} \oplus M^{\prime}$, with $L^{\prime}$ and $M^{\prime}$ ample line bundles on $X$. By what we have said above, $\varepsilon(Y, A)>1$, with $A=\operatorname{det}(\mathcal{E})=L^{\prime} \otimes M^{\prime}$. Moreover, the normal bundle $N$ is isomorphic to $L \oplus M$, where $L=L^{\prime} \mid Y$ and $M=M^{\prime} \mid Y$ (in particular, $L$ and $M$ are ample line bundles on $Y$ ). Then it is easy to compute the Segre classes of the normal bundle $N=\mathcal{E}_{Y}$. Namely, for every line bundle $\mathcal{L}$ on $Y$ and for every $i \geq 0$ one has $s_{i}(\mathcal{L})=(-1)^{i} c_{1}(\mathcal{L})^{\cdot i}$. Therefore for every integer $t \geq 0$ we get

$$
s_{t}(N)=s_{t}(L \oplus M)=\sum_{i=0}^{t} s_{i}(L) \cdot s_{t-i}(M)=(-1)^{t} \sum_{i=0}^{t} c_{1}(L)^{\cdot i} \cdot c_{1}(M)^{\cdot(t-i)} .
$$

Using (77) and (24) and taking into account that $y-k+t+2=t$ (because $\left.\operatorname{codim}_{X}(Y)=k-y=2\right)$ in our situation we obtain

$$
\delta_{\eta}(Y, A)=-\sum_{t=0}^{k-2}\left(\begin{array}{c}
k-2 \\
t
\end{array}\right) \eta^{t} \int_{Y} s_{t}(N) \cdot c_{1}\left(A_{Y}\right)^{\cdot(k-t-2)}
$$




$$
\begin{aligned}
& =\sum_{t=0}^{k-2}(-1)^{t+1}\left(\begin{array}{c}
k-2 \\
t
\end{array}\right) \eta^{t} \int_{Y} \sum_{i=0}^{t} c_{1}(L)^{\cdot i} \cdot c_{1}(M)^{\cdot(t-i)} \cdot c_{1}(L \otimes M)^{\cdot(k-t-2)} \\
& =\sum_{t=0}^{k-2}(-1)^{t+1}\left(\begin{array}{c}
k-2 \\
t
\end{array}\right) \eta^{t} \int_{Y}\left(c_{1}(L)+c_{1}(M)\right)^{\cdot(k-t-2)} \cdot \sum_{i=0}^{t} c_{1}(L)^{\cdot i} \cdot c_{1}(M)^{\cdot(t-i)}
\end{aligned}
$$

To proceed further we need the following elementary result of algebra.

Lemma 3.3 Let $B$ be a commutative unitary ring and let $b_{1}, b_{2} \in B$. Then for every integer $y \geq 1$ one has

$$
\sum_{t=0}^{y}(-1)^{t+1}\left(\begin{array}{l}
y \\
t
\end{array}\right)\left(b_{1}+b_{2}\right)^{y-t} \sum_{i=0}^{t} b_{1}^{i} b_{2}^{t-i}=\sum_{j=1}^{y-1} b_{1}^{y-j} b_{2}^{j} .
$$

Proof. Consider the polynomial ring $\mathbb{Z}\left[T_{1}, T_{2}\right]$ in two variables $T_{1}$ and $T_{2}$ over the ring of integers $\mathbb{Z}$. We claim that the identity

$$
\sum_{t=0}^{y}(-1)^{t+1}\left(\begin{array}{l}
y \\
t
\end{array}\right)\left(T_{1}+T_{2}\right)^{y-t} \sum_{i=0}^{t} T_{1}^{i} T_{2}^{t-i}=\sum_{j=1}^{y-1} T_{1}^{y-j} T_{2}^{j}
$$

holds true. Indeed, if we denote by $I$ the left hand side member of the identity to be proved, we have successively (in the fraction field $\mathbb{Q}\left(T_{1}, T_{2}\right)$ ):

$$
\begin{aligned}
I & =\sum_{t=0}^{y}(-1)^{t+1}\left(\begin{array}{c}
y \\
t
\end{array}\right)\left(T_{1}+T_{2}\right)^{y-t} \frac{T_{1}^{t+1}-T_{2}^{t+1}}{T_{1}-T_{2}} \\
& =\frac{1}{T_{1}-T_{2}} \sum_{t=0}^{y}(-1)^{t+1}\left(\begin{array}{c}
y \\
t
\end{array}\right)\left(T_{1}+T_{2}\right)^{y-t} T_{1}^{t+1}-\frac{1}{T_{1}-T_{2}} \sum_{t=0}^{y}(-1)^{t+1}\left(\begin{array}{c}
y \\
t
\end{array}\right)\left(T_{1}+T_{2}\right)^{y-t} T_{2}^{t+1} \\
& =\frac{-T_{1}}{T_{1}-T_{2}} \sum_{t=0}^{y}\left(\begin{array}{c}
y \\
t
\end{array}\right)\left(T_{1}+T_{2}\right)^{y-t}\left(-T_{1}\right)^{t}+\frac{T_{2}}{T_{1}-T_{2}} \sum_{t=0}^{y}\left(\begin{array}{c}
y \\
t
\end{array}\right)\left(T_{1}+T_{2}\right)^{y-t}\left(-T_{2}\right)^{t} \\
& =\frac{-T_{1}}{T_{1}-T_{2}}\left(\left(T_{1}+T_{2}\right)-T_{1}\right)^{y}+\frac{T_{2}}{T_{1}-T_{2}}\left(\left(T_{1}+T_{2}\right)-T_{2}\right)^{y} \\
& =\frac{T_{1} T_{2}\left(T_{1}^{y-1}-T_{2}^{y-1}\right)}{T_{1}-T_{2}}=T_{1} T_{2} \sum_{j=1}^{y-1} T_{1}^{y-j-1} T_{2}^{j-1}=\sum_{j=1}^{y-1} T_{1}^{y-j} T_{2}^{j},
\end{aligned}
$$

which proves our claim.

To prove the identity in general, consider the ring homomorphism

$$
\varphi: \mathbb{Z}\left[T_{1}, T_{2}\right] \rightarrow B
$$

such that $\varphi(n)=n \cdot 1_{B}$ and $\varphi\left(T_{i}\right)=b_{i}, i=1,2$. Then the conclusion follows from (25) because

$$
\begin{aligned}
\sum_{t=0}^{y}(-1)^{t+1}\left(\begin{array}{l}
y \\
t
\end{array}\right) & \left(b_{1}+b_{2}\right)^{y-t} \sum_{i=0}^{t} b_{1}^{i} b_{2}^{t-i}-\sum_{j=1}^{y-1} b_{1}^{y-j} b_{2}^{j} \\
= & \varphi\left(\sum_{t=0}^{y}(-1)^{t+1}\left(\begin{array}{c}
y \\
t
\end{array}\right)\left(T_{1}+T_{2}\right)^{y-t} \sum_{i=0}^{t} T_{1}^{i} T_{2}^{t-i}-\sum_{j=1}^{y-1} T_{1}^{y-j} T_{2}^{j}\right)=\varphi(0)=0
\end{aligned}
$$

Now we are ready to prove the following result. 
Proposition 3.4 Let $\mathcal{E}=L^{\prime} \oplus M^{\prime}$ be a decomposable ample vector bundle of rank 2 on a smooth projective variety $X$ of dimension $k \geq 3$, and let $s=(f, g) \in H^{0}(\mathcal{E})=H^{0}\left(L^{\prime} \oplus M^{\prime}\right)$ be a global section such that the zero locus $Y:=Z(s)$ is smooth of codimension 2 in $X$. Then $Y$ is Seshadri $A$-big, with $A:=L^{\prime} \otimes M^{\prime}$. In particular, if we fix a projective embedding $X \hookrightarrow \mathbb{P}^{n}$, every smooth complete intersection in $X$ of codimension 2 is Seshadri $\mathcal{O}_{X}(1)$-big.

Proof. Since we have seen above that the Seshadri constant $\varepsilon\left(Y, L^{\prime} \otimes M^{\prime}\right)$ is $>1$, it will be enough to show that $\delta_{1}(Y, A)>0$, where $A=L^{\prime} \otimes M^{\prime}$. To this end, in Lemma 3.3 take as $B$ the Chow ring $A(Y)$ of cycles of $Y$ modulo the rational equivalence, $y=\operatorname{dim}(Y)$, and put $b_{1}=c_{1}(L)$ and $b_{2}=c_{1}(M)$ (recall that $L=L^{\prime} \mid Y$ and $M=M^{\prime} \mid Y$ ). Then by Lemma 3.3 we get

$$
\left(\sum_{t=0}^{y}(-1)^{t+1}\left(\begin{array}{l}
y \\
t
\end{array}\right)\left(c_{1}(L)+c_{1}(M)\right)^{\cdot(y-t)}\right) \cdot\left(\sum_{i=0}^{t} c_{1}(L)^{\cdot i} \cdot c_{1}(M)^{\cdot(t-i)}\right)=\sum_{j=1}^{y-1} c_{1}(L)^{\cdot(y-j)} \cdot c_{1}(M)^{\cdot j},
$$

which implies

$$
\delta_{1}(Y, A)=\sum_{j=1}^{y-1} \int_{Y} c_{1}(L)^{\cdot(y-j)} \cdot c_{1}(M)^{\cdot j}>0,
$$

because $\int_{Y} c_{1}(L)^{\cdot(y-j)} \cdot c_{1}(M)^{\cdot j}>0$ for every $j=1, \ldots, y-1$ (since $L$ and $M$ are ample line bundles on $Y$ ).

To show the last statement take $L^{\prime}=\mathcal{O}_{X}\left(d_{1}\right)$ and $M^{\prime}=\mathcal{O}_{X}\left(d_{2}\right)$, with $d_{1}$ and $d_{2}$ positive integers, and use the argument above together with Remark 1.3.

Now let us consider the case when the vector bundle $\mathcal{E}$ is indecomposable. As above, we have $y=k-2$ and $\varepsilon(Y, A)>1$, so can take $\eta=1$. Taking into account of relations (10), (17), (18) and (19) and using the facts that $N=\mathcal{E}_{Y}=\mathcal{E} \otimes \mathcal{O}_{Y}$ and $A=\operatorname{det}(\mathcal{E})$ (whence $A_{Y}=\operatorname{det}\left(\mathcal{E}_{Y}\right)$ and $\left.c_{1}\left(\mathcal{E}_{Y}\right)=c_{1}\left(A_{Y}\right)\right)$, we find:

$$
\begin{aligned}
\delta(Y, A) & =\varepsilon(Y, A) \operatorname{deg}\left(\mathcal{E}_{Y}\right)-\operatorname{deg}\left(\mathcal{E}_{Y}\right)>0, \text { for }(k, y)=(3,1), \\
\delta_{1}(Y, A) & =\int_{Y} c_{2}\left(\mathcal{E}_{Y}\right), \text { for }(k, y)=(4,2), \\
\delta_{1}(Y, A) & =\int_{Y} c_{1}\left(\mathcal{E}_{Y}\right) \cdot c_{2}\left(\mathcal{E}_{Y}\right), \text { for }(k, y)=(5,3), \\
\delta_{1}(Y, A) & =\int_{Y} c_{2}\left(\mathcal{E}_{Y}\right) \cdot\left(c_{1}\left(\mathcal{E}_{Y}\right)^{\cdot 2}-c_{2}\left(\mathcal{E}_{Y}\right)\right), \text { for }(k, y)=(6,4), \\
\delta_{1}(Y, A) & =\int_{Y} c_{1}\left(\mathcal{E}_{Y}\right) \cdot c_{2}\left(\mathcal{E}_{Y}\right) \cdot\left(c_{1}\left(\mathcal{E}_{Y}\right)^{\cdot 2}-2 c_{2}\left(\mathcal{E}_{Y}\right)\right), \text { for }(k, y)=(7,5) .
\end{aligned}
$$

Now, as the vector bundle $\mathcal{E}$ is ample, the restriction $\mathcal{E}_{Y}$ is also ample. We claim that $\delta_{1}(Y, A)>0$ if $k \leq 6$. Indeed, if $(k, y)=(4,2)$, this follows from (27) and from a result of Kleiman [25] (see also Bloch and Gieseker [10] for a subsequent more general result) according to which $\int_{Y} c_{2}\left(\varepsilon_{Y}\right)>0$ when $Y$ is a surface.

If $(k, y)=(5,3)$ by (28) we have

$$
\begin{aligned}
\delta_{1}(Y, A) & =\int_{Y} c_{1}\left(\varepsilon_{Y}\right) \cdot c_{2}\left(\varepsilon_{Y}\right)=\int_{Y}\left|\begin{array}{ccc}
c_{2}\left(\varepsilon_{Y}\right) & 0 & 0 \\
1 & c_{1}\left(\mathcal{E}_{Y}\right) & c_{2}\left(\varepsilon_{Y}\right) \\
0 & 0 & 1
\end{array}\right| \\
& =\int_{Y}\left|\begin{array}{ccc}
c_{2} & c_{3} & c_{4} \\
c_{0} & c_{1} & c_{2} \\
c_{-2} & c_{-1} & c_{0}
\end{array}\right|\left(\varepsilon_{Y}\right),
\end{aligned}
$$


where the latter determinant is, in terminology of [15], the Schur polynomial (evaluated at $\mathcal{E}_{Y}$ ) associated to the partition $r \geq \lambda_{1} \geq \lambda_{2} \geq \lambda_{3} \geq 0$, whith $r=\operatorname{rank}\left(\mathcal{E}_{Y}\right)=2$ and $\left(\lambda_{1}, \lambda_{2}, \lambda_{3}\right)=$ $(2,1,0)$. This determinant is strictly positive by the main result of [15], or also [27, Theorem 8.3.9].

If $(k, y)=(6,4)$ by (29) we have

$$
\begin{aligned}
\delta_{1}(Y, A) & =\int_{Y} c_{2}\left(\mathcal{E}_{Y}\right) \cdot\left(c_{1}\left(\mathcal{E}_{Y}\right)^{-2}-c_{2}\left(\mathcal{E}_{Y}\right)\right) \\
& =\int_{Y}\left|\begin{array}{cccc}
c_{2}\left(\mathcal{E}_{Y}\right) & 0 & 0 & 0 \\
1 & c_{1}\left(\mathcal{E}_{Y}\right) & c_{2}\left(\mathcal{E}_{Y}\right) & 0 \\
0 & 1 & c_{1}\left(\mathcal{E}_{Y}\right) & c_{2}\left(\mathcal{E}_{Y}\right) \\
0 & 0 & 0 & 1
\end{array}\right|=\int_{Y}\left|\begin{array}{cccc}
c_{2} & c_{3} & c_{4} & c_{5} \\
c_{0} & c_{1} & c_{2} & c_{3} \\
c_{-1} & c_{0} & c_{1} & c_{2} \\
c_{-3} & c_{-2} & c_{-1} & c_{0}
\end{array}\right|\left(\mathcal{E}_{Y}\right),
\end{aligned}
$$

where the latter determinant is, in terminology of [15, the Schur polynomial (evaluated at $\left.\mathcal{E}_{Y}\right)$ associated to the partition $r \geq \lambda_{1} \geq \lambda_{2} \geq \lambda_{3} \geq \lambda_{4} \geq 0$, with $r=\operatorname{rank}\left(\mathcal{E}_{Y}\right)=2$ and $\left(\lambda_{1}, \lambda_{2}, \lambda_{3}, \lambda_{4}\right)=(2,1,1,0)$. Again, the determinant is strictly positive because $\mathcal{E}_{Y}$ is ample, by the main result of [15], see also [27, Theorem 8.3.9].

Assume now $(k, y)=(7,5)$. The polynomial $c_{1} \cdot c_{2} \cdot\left(c_{1}^{2}-2 c_{2}\right)$ occurring in (30) is not positive (because $c_{1}^{2}-2 c_{2}$ is not a linear combination of the Schur polynomials $c_{1}^{2}-c_{2}$ and $c_{2}$, see [15]), whence $\delta_{1}(Y, A)$ is not always positive. Note incidently that the polynomial $c_{1}^{2}-2 c_{2}$ has a rather interesting story, see [27, Example 8.3.11, p. 121], II, and also [16, p. 418].

Putting things together and using Lemma 1.9 together with formula (30), we proved the following result (the case $(k, y)=(3,1)$ of Proposition 3.5 was treated in 28]).

Proposition 3.5 Let $\mathcal{E}$ be an ample vector bundle of rank 2 on a projective manifold $X$ of dimension $k$, with $3 \leq k \leq 6$, and let $s \in H^{0}(X, \mathcal{E})$ be a global section of $\mathcal{E}$ such that the zero locus $Y:=Z(s)$ is a smooth 2-codimensional submanifold of $X$. Then $Y$ is Seshadri A-big in $X$, where $A:=\operatorname{det}(\mathcal{E})$. If $(k, y)=(7,5)$ then $Y$ is Seshadri $A$-big if $\int_{Y} c_{1}\left(\mathcal{E}_{Y}\right) \cdot c_{2}\left(\mathcal{E}_{Y}\right)$. $\left(c_{1}\left(\mathcal{E}_{Y}\right)^{\cdot 2}-2 c_{2}\left(\mathcal{E}_{Y}\right)\right)>0$.

3.6 Submanifolds of $\mathbb{P}^{k}$ and their Chern classes. In order to give some relevant examples of Seshadri $\mathcal{O}_{\mathbb{P}^{k}}$ (1)-big submanifolds $Y$ of $X=\mathbb{P}^{k}$ of dimension $y \geq 2$, we need some generalities regarding Chern classes which allow us to explicitly compute Chern classes of the normal bundle of some submanifolds of $\mathbb{P}^{k}$.

The restriction to $Y$ of the Euler sequence of $\mathbb{P}^{k}$,

$$
0 \rightarrow \mathcal{O}_{Y} \rightarrow \mathcal{O}_{Y}(1)^{\oplus k+1} \rightarrow T_{\mathbb{P} k} \mid Y \rightarrow 0,
$$

where $T_{\mathbb{P}^{k}}$ is the tangent bundle of $\mathbb{P}^{k}$, yields the equality of Chern polynomials (see [12, $\mathrm{p}$. 50])

$$
c_{t}\left(T_{\mathbb{P}^{k}} \mid Y\right)=c_{t}\left(\mathcal{O}_{Y}(1)^{\oplus k+1}\right) .
$$

By additivity, formula (31) can be rewritten as

$$
c_{t}\left(T_{\mathbb{P}^{k}} \mid Y\right)=\left(1+t c_{1}\left(\mathcal{O}_{Y}(1)\right)^{k+1} .\right.
$$

Taking Chern classes in the latter formula we get

$$
c_{i}\left(T_{\mathbb{P}^{k}} \mid Y\right)=\left(\begin{array}{c}
k+1 \\
i
\end{array}\right) c_{1}\left(\mathcal{O}_{Y}(1)\right)^{\cdot i}, i=0,1, \ldots, y .
$$

In particular, if $i=y$ we get $c_{y}\left(T_{\mathbb{P}^{k}} \mid Y\right)=\left(\begin{array}{c}k+1 \\ y\end{array}\right) \operatorname{deg}(Y)$. 
On the other hand, the normal sequence

$$
0 \rightarrow T_{Y} \rightarrow T_{\mathbb{P}^{k}} \mid Y \rightarrow N \rightarrow 0
$$

and the additivity of the Chern classes yield the equality

$$
c_{t}\left(T_{\mathbb{P}^{k}} \mid Y\right)=c_{t}\left(T_{Y}\right) \cdot c_{t}(N),
$$

where $N$ is the normal bundle of $Y$ in $\mathbb{P}^{k}$. The latter equality can be rewritten via (32) as

$$
\sum_{i=0}^{y}\left(\begin{array}{c}
k+1 \\
i
\end{array}\right) c_{1}\left(\mathcal{O}_{Y}(1)\right)^{\cdot i} t^{i}=\left(\sum_{i=0}^{y} c_{i}(Y) t^{i}\right) \cdot\left(\sum_{i=0}^{y} c_{i}(N) t^{i}\right)
$$

where $c_{i}(Y):=c_{i}\left(T_{Y}\right), i=0,1, \ldots, y$, are the Chern classes of $Y$.

3.7 Rational normal scrolls of dimension 2. Let $Y_{e}$ be the image in $\mathbb{P}^{e+3}$ of the geometrically ruled surface (or Segre-Hirzebruch surface) $\mathbb{F}_{e}:=\mathbb{P}\left(\mathcal{O}_{\mathbb{P}^{1}} \oplus \mathcal{O}_{\mathbb{P}^{1}}(-e)\right), e \geq 0$, embedded as a scroll in $\mathbb{P}^{e+3}$ via the complete linear system $\left|C_{0}+(e+1) F\right|$, where $C_{0}$ is the minimal section (with $C_{0}^{\cdot 2}=-e$ ) and $F$ is any fiber of the canonical projection $\pi: \mathbb{F}_{e} \rightarrow \mathbb{P}^{1}$ respectively (we follow here the notation as in Hartshorne 23]). Moreover, $Y_{e}$ is a surface of minimal degree $e+2$ in $\mathbb{P}^{e+3}$, whence by Example 1.7 we have

$$
\varepsilon\left(Y_{e}, \mathcal{O}_{\mathbb{P}^{e+3}}(1)\right)=\frac{1}{2} .
$$

Furthermore, $c_{1}\left(Y_{e}\right)=-K_{e}$ and $\int_{Y} c_{2}\left(Y_{e}\right)=\chi_{\mathrm{top}}\left(Y_{e}\right)=2-2 b_{1}+b_{2}=4$ (see 3, Chapter 5]), where $K_{e}$ is the canonical class of $Y_{e}, \chi_{\mathrm{top}}\left(Y_{e}\right)$ is the topological Euler characteristic of $Y_{e}$, and $b_{i}, i=1,2$, are the Betti numbers of $Y_{e}$. In our case, $b_{1}=0$ and $b_{2}=2$, so $\int_{Y_{e}} c_{2}\left(Y_{e}\right)=4$.

Let $N$ be the normal bundle of $Y_{e}$ in $\mathbb{P}^{e+3}$. Then the identity (33) gives

$$
c_{1}\left(\mathcal{O}_{Y_{e}}\left(-K_{e}\right)\right)+c_{1}(N)=(e+4) c_{1}\left(\mathcal{O}_{Y_{e}}(1)\right)=c_{1}\left(\mathcal{O}_{Y_{e}}(e+4)\right),
$$

and

$$
\int_{Y_{e}} c_{2}(N)-\int_{Y_{e}} c_{1}\left(\mathcal{O}_{Y_{e}}\left(K_{e}\right)\right) \cdot c_{1}(N)+4=\left(\begin{array}{c}
e+4 \\
2
\end{array}\right) \operatorname{deg}\left(Y_{e}\right)=\frac{(e+4)(e+3)(e+2)}{2} .
$$

Recalling that $\mathcal{O}_{Y_{e}}\left(K_{e}\right)=\mathcal{O}_{Y_{e}}\left(-2 C_{0}-(e+2) F\right)$ and $\mathcal{O}_{Y_{e}}(e+4)=\mathcal{O}_{Y_{e}}\left((e+4) C_{0}+(e+4)(e+1) F\right)$, from the first equality we get

$$
c_{1}(N)=c_{1}\left(\mathcal{O}_{Y_{e}}\left((e+2) C_{0}+\left(e^{2}+4 e+2\right) F\right)\right),
$$

while the second equality yields

$$
\int_{Y_{e}} c_{2}(N)=\frac{(e+4)(e+3)(e+2)}{2}-e^{2}-8 e-12 .
$$

Now using (35) and (36), we get

$$
\int_{Y_{e}}\left(c_{1}(N)^{\cdot 2}-c_{2}(N)\right)=\frac{e^{3}+9 e^{2}+22 e+16}{2} \text { and } \int_{Y_{e}} c_{1}(N) \cdot c_{1}\left(\mathcal{O}_{Y_{e}}(1)\right)=e^{2}+5 e+4 .
$$

Therefore taking into account of (34) and (11) (with $k=e+3$ and $\operatorname{deg}\left(Y_{e}\right)=e+2$ ) we find, for every $e \geq 0$,

$$
\delta_{\eta}\left(Y_{e}, \mathcal{O}_{\mathbb{P}^{e+3}}(1)\right)=\frac{\eta^{e-1}}{2}\left(-\left(e^{3}+9 e^{2}+22 e+16\right) \eta^{2}+2(e+1)\left(e^{2}+5 e+4\right) \eta-e(e+1)(e+2)\right) .
$$


Letting $\eta=\varepsilon\left(Y_{e}, \mathcal{O}_{\mathbb{P}^{e+3}}(1)\right)=\frac{1}{2}$, after a straightforward computation the latter equality yields:

$$
\delta\left(Y_{e}, \mathcal{O}_{\mathbb{P}^{e+3}}(1)\right)=\frac{e}{2^{e+2}}\left(-e^{2}+3 e+6\right) .
$$

We can now prove the following:

Proposition 3.8 The image $Y_{e}$ in $\mathbb{P}^{e+3}$ of the rational normal scroll $\mathbb{F}_{e}$ is Seshadri $\mathcal{O}_{\mathbb{P} e+3}(1)$ big if and only if $0 \leq e \leq 4$.

Proof. From (38) it follows that $\delta\left(Y_{e}, \mathcal{O}_{\mathbb{P} e+3}(1)\right)>0$ if and only if $0 \leq e \leq 4$. It follows that $Y_{e}$ is Seshadri $\mathcal{O}_{\mathbb{P} e+3}(1)$-big if $0 \leq e \leq 4$.

On the other hand, racalling (37), consider the function $f: \mathbb{R} \rightarrow \mathbb{R}$ defined by

$$
f(x)=-\left(e^{3}+9 e^{2}+22 e+16\right) x^{2}+2(e+1)\left(e^{2}+5 e+4\right) x-e(e+1)(e+2) .
$$

Then $f$ is increasing on the interval $\left(0, \varepsilon\left(Y_{e}, \mathcal{O}_{\mathbb{P}^{e}+3}(1)\right)\right)=\left(0, \frac{1}{2}\right)$ if $e \geq 2$. Indeed, the function $f$ assumes the maximum value on $\mathbb{R}$ for

$$
x=\frac{(e+1)\left(e^{2}+5 e+4\right)}{e^{3}+9 e^{2}+22 e+16}=\frac{e^{3}+6 e^{2}+9 e+4}{e^{3}+9 e^{2}+22 e+16},
$$

and, moreover (as it is easily checked),

$$
\frac{e^{3}+6 e^{2}+9 e+4}{e^{3}+9 e^{2}+22 e+16}>\frac{1}{2} \quad \text { if } e \geq 2 .
$$

This implies that for $e \geq 2$ the function $f$ is increasing on the interval $\left(0, \frac{1}{2}\right)$. It follows that if $e \geq 2$ the surface $Y_{e}$ is Seshadri $\mathcal{O}_{\mathbb{P}^{e+3}}(1)$-big in $\mathbb{P}^{e+3}$ if and only if $\delta\left(Y_{e}, \mathcal{O}_{\mathbb{P}^{e+3}}(1)\right)>0$, or else (by the remark made at the beginning), only if $e \leq 4$.

3.9 The Veronese surface in $\mathbb{P}^{5}$ and its projection in $\mathbb{P}^{4}$. The last example of smooth surface of minimal degree is the Veronese surface $Y$ in $\mathbb{P}^{5}$, i.e., the image of the Veronese embedding $v_{2}: \mathbb{P}^{2} \hookrightarrow \mathbb{P}^{5}$. By Example 1.7, the Seshadri constant is $\varepsilon\left(Y, \mathcal{O}_{\mathbb{P}^{5}}(1)\right)=\frac{1}{2}$ whence, by formula (11),

$$
\begin{aligned}
\delta\left(Y, \mathcal{O}_{\mathbb{P}^{5}}(1)\right) & =-\frac{1}{8} \int_{Y}\left(c_{1}(N)^{\cdot 2}-c_{2}(N)\right)+\frac{3}{4} \int_{Y} c_{1}(N) \cdot c_{1}\left(\mathcal{O}_{Y}(1)\right)-\frac{3}{2} \operatorname{deg}(Y) \\
& =-\frac{1}{8} \int_{Y}\left(c_{1}(N)^{\cdot 2}-c_{2}(N)\right)+\frac{3}{4} \int_{Y} c_{1}(N) \cdot c_{1}\left(\mathcal{O}_{\mathbb{P}^{2}}(2)\right)-6 .
\end{aligned}
$$

Computing the Chern classes of the normal bundle $N$ by using (33) (as in the case of the surface scrolls), we immediately find

$$
c_{1}(N)=c_{1}\left(\mathcal{O}_{\mathbb{P}^{2}}(9)\right) \text { and } \int_{Y} c_{2}(N)=30 .
$$

Putting things together we get

$$
\delta\left(Y, \mathcal{O}_{\mathbb{P}^{5}}(1)\right)=-\frac{51}{8}+\frac{27}{2}-6=\frac{9}{8}>0 .
$$

Consider now the linear projection in $\mathbb{P}^{4}$ of the Veronese surface $Y=v_{2}\left(\mathbb{P}^{2}\right)$ from a general point of $\mathbb{P}^{5}$. It is a surface $Y^{\prime} \cong \mathbb{P}^{2}$ in $\mathbb{P}^{4}$ such that $\mathcal{O}_{Y^{\prime}}(1)=\mathcal{O}_{\mathbb{P}^{2}}(2)$. Let $N^{\prime}:=N_{Y^{\prime} \mid \mathbb{P}^{4}}$ be the 
normal bundle of $Y^{\prime}$ in $\mathbb{P}^{4}$. Then it is known that the homogeneous ideal $\mathcal{J}_{+}\left(Y^{\prime}\right)$ of $Y^{\prime}$ in $\mathbb{P}^{4}$ is generated by homogeneous polynomials of degree 3. (We thank John Abbott for providing us an explicit set of generators of degree 3 of $\mathcal{J}_{+}\left(Y^{\prime}\right)$ using CoCoA-4.7.) Then by Corollary 1.6 in [5], $\varepsilon\left(Y^{\prime}, \mathcal{O}_{\mathbb{P}^{4}}(1)\right) \geq \frac{1}{3}$. Therefore for $\eta \in\left(0, \frac{1}{3}\right]$ we have

$$
\begin{aligned}
\delta_{\eta}\left(Y^{\prime}, \mathcal{O}_{\mathbb{P}^{4}}(1)\right) & =-\eta^{2} \int_{Y^{\prime}}\left(c_{1}\left(N^{\prime}\right)^{-2}-c_{2}\left(N^{\prime}\right)\right)+2 \eta \int_{Y^{\prime}} c_{1}\left(N^{\prime}\right) \cdot c_{1}\left(\mathcal{O}_{Y^{\prime}}(1)\right)-\operatorname{deg}\left(Y^{\prime}\right) \\
& =-\eta^{2} \int_{Y^{\prime}}\left(c_{1}\left(N^{\prime}\right)^{-2}-c_{2}\left(N^{\prime}\right)\right)+2 \eta \int_{Y^{\prime}} c_{1}\left(N^{\prime}\right) \cdot c_{1}\left(\mathcal{O}_{\mathbb{P}^{2}}(2)\right)-4 .
\end{aligned}
$$

Computing as above the Chern classes of $N^{\prime}$ we find

$$
c_{1}\left(N^{\prime}\right)=c_{1}\left(\mathcal{O}_{\mathbb{P}^{2}}(7)\right) \text { and } \int_{Y^{\prime}} c_{2}\left(N^{\prime}\right)=16,
$$

whence

$$
\delta_{\eta}\left(Y^{\prime}, \mathcal{O}_{\mathbb{P}^{4}}(1)\right)=-33 \eta^{2}+28 \eta-4 .
$$

In particular,

$$
\delta_{\frac{1}{3}}\left(Y^{\prime}, \mathcal{O}_{\mathbb{P}^{4}}(1)\right)=-\frac{33}{9}+\frac{28}{3}-4=\frac{5}{3}>0 .
$$

Summarizing, (39) and (40) yield the following result.

Proposition 3.10 The Veronese surface (i.e., the image $Y$ of the Veronese embedding $\mathbb{P}^{2} \hookrightarrow$ $\left.\mathbb{P}^{5}\right)$ is Seshadri $\mathcal{O}_{\mathbb{P}^{5}}(1)$-big in $\mathbb{P}^{5}$. Let $Y^{\prime} \cong \mathbb{P}^{2}$ be the image of $Y$ via the linear projection in $\mathbb{P}^{4}$ of $Y$ from a general point of $\mathbb{P}^{5}$. Then $Y^{\prime}$ is Seshadri $\mathcal{O}_{\mathbb{P}^{4}}(1)$-big in $\mathbb{P}^{4}$.

3.11 Some Segre embeddings. We prove here the $\mathcal{O}(1)$-bigness of some Segre embeddings.

First, consider the Segre embedding $i_{5}: \mathbb{P}^{2} \times \mathbb{P}^{1} \hookrightarrow \mathbb{P}^{5}$, and set $Y:=i_{5}\left(\mathbb{P}^{2} \times \mathbb{P}^{1}\right)$. By Example 1.7 $\varepsilon\left(Y, \mathcal{O}_{\mathbb{P}^{5}}(1)\right)=\frac{1}{2}$. Since $\operatorname{codim}_{\mathbb{P}^{5}}(Y)=2$ we can apply (18) to get

$$
\begin{aligned}
\delta_{\eta}\left(Y, \mathcal{O}_{\mathbb{P}^{5}}(1)\right)= & -3+3 \eta \int_{Y} c_{1}(N) \cdot c_{1}\left(\mathcal{O}_{Y}(1)\right)^{\cdot 2} \\
& -3 \eta^{2}\left(c_{1}(N)^{\cdot 2}-c_{2}(N)\right) \cdot c_{1}\left(\mathcal{O}_{Y}(1)\right)-\eta^{3} \int_{Y}\left(2 c_{1}(N) \cdot c_{2}(N)-c_{1}(N)^{\cdot 3}\right),
\end{aligned}
$$

where $\delta_{\eta}:=\delta_{\eta}\left(Y, \mathcal{O}_{\mathbb{P}^{5}}(1)\right)$. The equation (33) reads in this case

$$
\left(1+c_{1}\left(\mathcal{O}_{Y}(1)\right) t\right)^{6}=\left(1+c_{1}(Y) t+c_{2}(Y) t^{2}+c_{3}(Y) t^{3}\right) \cdot\left(1+c_{1}(N) t+c_{2}(N) t^{2}\right) .
$$

Identifying the coefficients of $t$ and using $c_{1}(Y)=-c_{1}\left(K_{Y}\right)$, we get

$$
c_{1}(N)=c_{1}\left(K_{Y}\right)+6 c_{1}\left(\mathcal{O}_{Y}(1)\right)=c_{1}\left(\mathcal{O}_{Y}(6)\right)+c_{1}\left(K_{Y}\right),
$$

whence

$$
c_{1}(N)=c_{1}(\mathcal{O}(3,4)),
$$

taking into account that $\mathcal{O}_{Y}(1)=\mathcal{O}(1,1)$ and $K_{Y}=\mathcal{O}(-3,-2)$, where as usual we set $\mathcal{O}(a, b):=$ $p_{1}^{*}\left(\mathcal{O}_{\mathbb{P}^{2}}(a)\right) \otimes p_{2}^{*}\left(\mathcal{O}_{\mathbb{P}^{1}}(b)\right)$, with $p_{1}$ and $p_{2}$ the canonical projections of $\mathbb{P}^{2} \times \mathbb{P}^{1}$. Identifying the coefficients of $t^{2}$ in (42) we also get

$$
c_{2}(N)-c_{1}\left(K_{Y}\right) \cdot c_{1}(N)+c_{2}\left(T_{Y}\right)=15 c_{1}\left(\mathcal{O}_{Y}(1)\right)^{2} .
$$


Using (43) and the fact that

$$
T_{Y}=p_{1}^{*}\left(T_{\mathbb{P}^{2}}\right) \oplus p_{2}^{*}\left(T_{\mathbb{P}^{1}}\right)
$$

we find

$$
\left.c_{t}\left(T_{Y}\right)\right)=c_{t}\left(p_{1}^{*}\left(T_{\mathbb{P}^{2}}\right)\right) \cdot c_{t}\left(p_{2}^{*}\left(T_{\mathbb{P}^{1}}\right)\right)=p_{1}^{*}\left(c_{t}\left(T_{\mathbb{P}^{2}}\right)\right) \cdot p_{2}^{*}\left(c_{t}\left(T_{\mathbb{P}^{1}}\right)\right) .
$$

Since $c_{t}\left(T_{\mathbb{P}^{2}}\right)=1+c_{1}\left(\mathcal{O}_{\mathbb{P}^{2}}(3)\right) t+3 x t^{2}$, with $x \in \mathbb{P}^{2}$ and $c_{t}\left(T_{\mathbb{P}^{1}}\right)=1+c_{1}\left(\mathcal{O}_{\mathbb{P}^{1}}(2)\right) t$, we get

$$
c_{t}\left(T_{Y}\right)=\left(1+c_{1}(\mathcal{O}(3,0)) t+3\left(x \times \mathbb{P}^{1}\right) t^{2}\right) \cdot\left(1+c_{1}(\mathcal{O}(0,2) t) .\right.
$$

Identifying the coefficients of $t^{2}$ in this latter identity yields

$$
c_{2}\left(T_{Y}\right)=3\left(x \times \mathbb{P}^{1}\right)+c_{1}(\mathcal{O}(3,0)) \cdot c_{1}(\mathcal{O}(0,2))=3\left(x \times \mathbb{P}^{1}\right)+6(\ell \times y),
$$

where $\ell$ is a line of $\mathbb{P}^{2}$ and $y \in \mathbb{P}^{1}$. Substituting (45) in (44) and using the obvious formula

$$
c_{1}(\mathcal{O}(a, b)) \cdot c_{1}\left(\mathcal{O}\left(a^{\prime}, b^{\prime}\right)\right)=a a^{\prime}\left(x \times \mathbb{P}^{1}\right)+\left(a b^{\prime}+a^{\prime} b\right)(\ell \times y),
$$

we get

$$
c_{2}(N)=3\left(x \times \mathbb{P}^{1}\right)+6(\ell \times y), \text { with } x \in \mathbb{P}^{2}, y \in \mathbb{P}^{1} \text {, and } \ell \text { a line in } \mathbb{P}^{2} .
$$

Now we are ready to compute $\delta_{\eta}\left(Y, \mathcal{O}_{\mathbb{P}^{5}}(1)\right)$. Using repeatedly (43), (477) and (46) we have:

$$
\begin{aligned}
\int_{Y} c_{1}(N) \cdot c_{1}\left(\mathcal{O}_{Y}(1)\right)^{-2}= & \int_{Y} c_{1}(\mathcal{O}(3,4)) \cdot c_{1}(\mathcal{O}(1,1)) \cdot c_{1}(\mathcal{O}(1,1)) \\
= & \int_{Y} c_{1}(\mathcal{O}(3,4)) \cdot\left[\left(x \times \mathbb{P}^{1}\right)+2(\ell \times y)\right]=10 . \\
\int_{Y}\left(c_{1}(N)^{\cdot 2}-c_{2}(N)\right) \cdot c_{1}\left(\mathcal{O}_{Y}(1)\right)= & \int_{Y} c_{1}(\mathcal{O}(3,4)) \cdot c_{1}(\mathcal{O}(3,4)) \cdot c_{1}(\mathcal{O}(1,1)) \\
& -\int_{Y}\left[3\left(x \times \mathbb{P}^{1}\right)+6(\ell \times y)\right] \cdot c_{1}(\mathcal{O}(1,1))=24 . \\
\int_{Y}\left(2 c_{1}(N) \cdot c_{2}(N)-c_{1}(N)^{\cdot 3}\right)= & 2 \int_{Y} c_{1}(\mathcal{O}(3,4)) \cdot\left[3\left(x \times \mathbb{P}^{1}\right)+6(\ell \times y)\right] \\
& -\int_{Y} c_{1}(\mathcal{O}(3,4)) \cdot\left[9\left(x \times \mathbb{P}^{1}\right)+24(\ell \times y)\right]=-48 .
\end{aligned}
$$

Substituting these equalities in (41) we find

$$
\delta_{\eta}\left(Y, \mathcal{O}_{\mathbb{P} 5}(1)\right)=48 \eta^{3}-72 \eta^{2}+30 \eta-3 .
$$

Taking $\eta=\frac{1}{3} \in\left(0, \frac{1}{2}\right)$ we get

$$
\delta_{\frac{1}{3}}\left(Y, \mathcal{O}_{\mathbb{P}^{5}}(1)\right)=\frac{48}{27}-\frac{72}{9}+\frac{30}{3}-3=\frac{16}{9}-8+10-3=\frac{16}{9}-1=\frac{7}{9}>0 .
$$

Note that $\delta\left(Y, \mathcal{O}_{\mathbb{P}^{5}}(1)\right)=\delta_{\frac{1}{2}}\left(Y, \mathcal{O}_{\mathbb{P}^{5}}(1)\right)=0$. (Using this and the fact that $f^{\prime}\left(\frac{1}{2}\right)<0$ we also deduce that $f(\eta)>0$ for every $\eta<\frac{1}{2}$ which is close to $\frac{1}{2}$, where $f(\eta)=48 \eta^{3}-72 \eta^{2}+30 \eta-3$.) Therefore $Y:=i_{5}\left(\mathbb{P}^{2} \times \mathbb{P}^{1}\right)$ is $\mathcal{O}_{\mathbb{P}^{5}}(1)$-big.

Next, consider the Segre embedding $i_{7}: \mathbb{P}^{3} \times \mathbb{P}^{1} \hookrightarrow \mathbb{P}^{7}$, and set $Y:=i_{7}\left(\mathbb{P}^{3} \times \mathbb{P}^{1}\right)$. By Example 1.7 again, $\varepsilon\left(Y, \mathcal{O}_{\mathbb{P}^{7}}(1)\right)=\frac{1}{2}$. The computations are completely similar as in the previous case. Specifically, as it is easily seen, we have $c_{1}\left(\mathcal{O}_{Y}(1)\right)=\left(P \times \mathbb{P}^{1}\right)+\left(\mathbb{P}^{3} \times y\right)$, $c_{1}(Y)=4\left(P \times \mathbb{P}^{1}\right)+2\left(\mathbb{P}^{3} \times y\right), c_{2}(Y)=6\left(\ell \times \mathbb{P}^{1}\right)+8(P \times y), c_{3}(Y)=4\left(x \times \mathbb{P}^{1}\right)+12(\ell \times y)$, and 
$c_{4}(Y)=8(x \times y)$, where $x \in \mathbb{P}^{3}$ and $y \in \mathbb{P}^{1}$ are points, $\ell$ is a line in $\mathbb{P}^{3}$ and $P$ is a plane in $\mathbb{P}^{3}$. Using equation (33) to compute the Chern classes of $N$ we find:

$$
\begin{aligned}
& c_{1}(N)=4\left(P \times \mathbb{P}^{1}\right)+6\left(\mathbb{P}^{3} \times y\right), \\
& c_{2}(N)=6\left(\ell \times \mathbb{P}^{1}\right)+16(P \times y), \\
& c_{3}(N)=4\left(x \times \mathbb{P}^{1}\right)+12(\ell \times y) .
\end{aligned}
$$

Using these formulae, (7), (15) and (16) we get

$$
\delta_{\eta}\left(Y, \mathcal{O}_{\mathbb{P}^{7}}(1)\right)=20 \eta\left(-1+9 \eta-26 \eta^{2}+30 \eta^{3}-12 \eta^{4}\right) .
$$

Setting $f(\eta)=-1+9 \eta-26 \eta^{2}+30 \eta^{3}-12 \eta^{4}$, we have

$$
\delta\left(Y, \mathcal{O}_{\mathbb{P}^{7}}(1)\right)=\delta_{\frac{1}{2}}\left(Y, \mathcal{O}_{\mathbb{P}^{7}}(1)\right)=10 f\left(\frac{1}{2}\right)=0, \text { but } \delta_{\frac{1}{3}}\left(Y, \mathcal{O}_{\mathbb{P}^{7}}(1)\right)=\frac{20}{3} f\left(\frac{1}{3}\right)=\frac{20}{3} \times \frac{2}{27}>0 .
$$

Therefore $Y=i_{7}\left(\mathbb{P}^{3} \times \mathbb{P}^{1}\right)$ is $\mathcal{O}_{\mathbb{P}^{7}}(1)$-big.

Finally, consider at the Segre embedding $i_{8}: \mathbb{P}^{2} \times \mathbb{P}^{2} \hookrightarrow \mathbb{P}^{8}$, and set $Y:=i_{8}\left(\mathbb{P}^{2} \times \mathbb{P}^{2}\right)$. In this case $Y=i\left(\mathbb{P}^{2} \times \mathbb{P}^{2}\right)$ is no more a subvariety of minimal degree because $\operatorname{deg}(Y)=6$ and $\operatorname{codim}_{\mathbb{P} 8}(Y)=4$. However, since $Y$ is not a linear subspace of $\mathbb{P}^{8}$ and $Y$ is still defined scheme-theoretically by quadratic equations, by Example 1.7] we still have $\varepsilon\left(Y, \mathcal{O}_{\mathbb{P}^{8}}(1)\right)=\frac{1}{2}$. It is immediate to see that $c_{1}\left(\mathcal{O}_{Y}(1)\right)=\left(\ell \times \mathbb{P}^{2}\right)+\left(\mathbb{P}^{2} \times \ell^{\prime}\right), c_{1}(Y)=3\left(\ell \times \mathbb{P}^{2}\right)+3\left(\mathbb{P}^{2} \times \ell^{\prime}\right)$, $c_{2}(Y)=3\left(x \times \mathbb{P}^{2}\right)+9\left(\ell \times \ell^{\prime}\right)+3\left(\mathbb{P}^{2} \times y\right), c_{3}(Y)=9(\ell \times y)+9\left(x \times \ell^{\prime}\right)$, and $c_{4}(Y)=9(x \times y)$, where $x, y \in \mathbb{P}^{2}$ are points and $\ell$ and $\ell^{\prime}$ are lines in $\mathbb{P}^{2}$. Using again (33) and proceeding similarly as above we find for the Chern classes of $N$ the expressions:

$$
\begin{aligned}
& c_{1}(N)=6\left(\ell \times \mathbb{P}^{2}\right)+6\left(\mathbb{P}^{2} \times \ell^{\prime}\right), \\
& c_{2}(N)=15\left(x \times \mathbb{P}^{2}\right)+27\left(\ell \times \ell^{\prime}\right)+15\left(\mathbb{P}^{2} \times y\right), \\
& c_{3}(N)=45(\ell \times y)+45\left(x \times \ell^{\prime}\right), \\
& c_{4}(N)=36(x \times y) .
\end{aligned}
$$

Now using (77), (15) and (16) together with the above relations, we get (for $\left.\eta \in\left(0, \frac{1}{2}\right)\right)$ :

$$
\delta_{\eta}\left(Y, \mathcal{O}_{\mathbb{P}^{8}}(1)\right)=9 \eta^{2}\left(-10+80 \eta-220 \eta^{2}+252 \eta^{3}-103 \eta^{4}\right)=9 \eta^{2} f(\eta),
$$

where $f(\eta):=-10+80 \eta-220 \eta^{2}+252 \eta^{3}-103 \eta^{4}$. Therefore $f\left(\frac{1}{2}\right)=-10+40-55+31.5-6.4375=$ $71.5-71.4375>0$, whence $\delta\left(Y, \mathcal{O}_{\mathbb{P}^{8}}(1)\right)=\delta_{\frac{1}{2}}\left(Y, \mathcal{O}_{\mathbb{P}^{8}}(1)\right)>0$. Thus $Y=i_{8}\left(\mathbb{P}^{2} \times \mathbb{P}^{2}\right)$ is $\mathcal{O}_{\mathbb{P}^{8}}(1)$ big.

Summing up we proved the following:

Proposition 3.12 The images of the Segre embeddings

$$
i_{5}: \mathbb{P}^{2} \times \mathbb{P}^{1} \hookrightarrow \mathbb{P}^{5}, \quad i_{7}: \mathbb{P}^{3} \times \mathbb{P}^{1} \hookrightarrow \mathbb{P}^{7} \quad \text { and } \quad i_{8}: \mathbb{P}^{2} \times \mathbb{P}^{2} \hookrightarrow \mathbb{P}^{8}
$$

are Seshadri $\mathcal{O}_{\mathbb{P}^{k}}(1)$-big, with $k=5, k=7$ and $k=8$ respectively.

Remark 3.13 The examples of Segre embeddings discussed above are especially interesting. Indeed, although the rational normal scrolls $\mathbb{F}_{e} \hookrightarrow \mathbb{P}^{e+3}$ are all set-theoretic (but not schemetheoretic) complete intersections in $\mathbb{P}^{e+3}$ (see [33], or also [6]), the product $\mathbb{P}^{2} \times \mathbb{P}^{1}$ (respectively $\mathbb{P}^{3} \times \mathbb{P}^{1}$ ) is not set-theoretic complete intersection in $\mathbb{P}^{5}$ (respectively in $\mathbb{P}^{7}$ ). In fact, $\mathbb{P}^{2} \times \mathbb{P}^{1}$ (respectively $\mathbb{P}^{3} \times \mathbb{P}^{1}$ ) is not even the zero locus of a section of an ample rank 2 vector bundle on $\mathbb{P}^{5}$ (respectively of an ample rank 3 vector bundle on $\mathbb{P}^{7}$ ), see [26] for $\mathbb{P}^{2} \times \mathbb{P}^{1}$ and [6. Corollary 4.5] for $\mathbb{P}^{3} \times \mathbb{P}^{1}$. 
3.14 An elliptic surface. The next result provides an example of an irregular surface $Y$ in $\mathbb{P}^{5}$ which is Seshadri $\mathcal{O}_{\mathbb{P}^{5}}(1)$-big.

Proposition 3.15 Consider the geometrically ruled elliptic surface $Y=C \times \mathbb{P}^{1}$, with $C$ a smooth elliptic plane curve, embedded in $\mathbb{P}^{5}$ via the composition $C \times \mathbb{P}^{1} \hookrightarrow \mathbb{P}^{2} \times \mathbb{P}^{1} \hookrightarrow \mathbb{P}^{5}$, where the second inclusion is the Segre embedding of $\mathbb{P}^{2} \times \mathbb{P}^{1}$. Then $Y$ is Seshadri $\mathcal{O}_{\mathbb{P}^{5}}(1)$-big in $\mathbb{P}^{5}$.

Proof. Assume that the elliptic curve $C$ is embedded in $\mathbb{P}^{2}$ as a cubic curve of equation $F\left(x_{0}, x_{1}, x_{2}\right)=0$. If $\left[y_{0}, y_{1}\right] \in \mathbb{P}^{1}$ then the homogeneous coordinates of a point of $\mathbb{P}^{5}$ are

$$
z_{i j}=x_{i} y_{j}, \text { with } i=0,1,2 \text { and } j=0,1 .
$$

Thus $Y$ becomes an elliptic scroll in $\mathbb{P}^{5}$. Then by [30] the homogeneous ideal of $Y$ in $\mathbb{P}^{5}$ is generated by the seven homogeneous polynomials $z_{10} z_{21}-z_{20} z_{11}, z_{20} z_{01}-z_{00} z_{21}, z_{00} z_{11}-z_{10} z_{01}$ and

$$
\sum_{i=0}^{2} z_{i s} \frac{\partial F}{\partial x_{i}}\left(z_{0 t}, z_{1 t}, z_{2 t}\right), \text { where } 0 \leq s, t \leq 1 .
$$

In particular, $Y$ is defined by $\mathcal{O}_{\mathbb{P}^{5}}(1)$ in degree 3 in the terminology of $[5$. Then from $[5$, Corollary 1.6] it follows that $\varepsilon\left(Y, \mathcal{O}_{\mathbb{P}^{5}}(1)\right) \geq \frac{1}{3}$.

In our situation we have $\mathcal{O}_{Y}(1)=p_{1}^{*}(L) \otimes p_{2}^{*}\left(\mathcal{O}_{\mathbb{P}^{1}}(1)\right)$, where $L=\mathcal{O}_{C}(1)$ is a line bundle of degree 3 on $C$, and $\mathcal{O}_{Y}\left(K_{Y}\right)=p_{2}^{*}\left(\mathcal{O}_{\mathbb{P}^{1}}(-2)\right.$. Using relation (33) and computing as in the proof of Proposition 3.10, we find:

$$
\operatorname{deg}(Y)=\mathcal{O}_{Y}(1)^{\cdot 2}=6, \quad c_{1}(N)=c_{1}\left(p_{1}^{*}\left(L^{\otimes 6}\right) \otimes p_{2}^{*}\left(\mathcal{O}_{\mathbb{P}^{1}}(4)\right), \quad c_{2}(Y)=0, \quad \text { and } \quad \int_{Y} c_{2}(N)=54 .\right.
$$

These formulae yield

$$
\int_{Y}\left(c_{1}(N)^{\cdot 2}-c_{2}(N)\right)=90 \text { and } \int_{Y} c_{1}(N) \cdot c_{1}\left(\mathcal{O}_{Y}(1)\right)=30 .
$$

Therefore by (12) we find

$$
\delta_{\eta}\left(Y, \mathcal{O}_{\mathbb{P}^{5}}(1)\right)=\eta\left(-90 \eta^{2}+90 \eta-18\right) .
$$

Taking $\eta=\frac{1}{3}$ we finally get $\delta_{\frac{1}{3}}\left(Y, \mathcal{O}_{\mathbb{P}^{5}}(1)\right)=\frac{-10+30-18}{3}=\frac{2}{3}>0$.

The following general result (which was noticed by Paoletti in 28 when $(k, y)=(3,1)$ ) allows one to produce many new examples of Seshadri $A$-big or $A$-ample submanifolds, starting from some known ones.

Proposition 3.16 Let $f: X^{\prime} \rightarrow X$ be a finite surjective morphism between smooth projective varieties. Let $A$ be an ample line bundle and set $A^{\prime}:=f^{*}(A)$. Let $Y$ be a smooth connected projective subvariety of $X$ and assume that $Y^{\prime}:=f^{-1}(Y)$ is smooth and connected. Then $\varepsilon\left(Y^{\prime}, A^{\prime}\right)=\varepsilon(Y, A)=: \varepsilon$ and $\delta_{\eta}\left(Y^{\prime}, A^{\prime}\right)=\operatorname{deg}(f) \delta_{\eta}(Y, A)$ for every $\eta \in(0, \varepsilon)$. In particular, $Y$ is Seshadri A-big (respectively A-ample) if and only if $Y^{\prime}$ is Seshadri $A^{\prime}$-big (respectively $A^{\prime}$-ample).

Proof. Denote by $\mathcal{J}_{Y}$ the sheaf of ideals of $Y$ in $\mathcal{O}_{X}$. By a general result (see e.g. [17, IV, (17.3.5)]), as $f: X^{\prime} \rightarrow X$ is a finite surjective morphism between smooth varieties, $f$ is a flat morphism. Therefore the canonical map $f^{*}\left(\mathcal{J}_{Y}\right) \rightarrow f^{*}\left(\mathcal{O}_{X}\right)=\mathcal{O}_{X^{\prime}}$ is injective. It follows that $\mathcal{J}_{Y^{\prime}} \cong f^{*}\left(\mathcal{J}_{Y}\right)$, where $\mathcal{J}_{Y^{\prime}}$ is the sheaf of ideals of $Y^{\prime}=f^{-1}(Y)$. This shows that the blowing 
up $X_{Y^{\prime}}^{\prime}$ of $X^{\prime}$ along $Y^{\prime}$ identifies with $\operatorname{Proj}\left(f^{*}\left(\oplus_{i=0}^{\infty} J_{Y}^{i}\right)\right)$ which, by [17, II, Proposition 3.5.3], coincides with the fibered product $\operatorname{Proj}\left(\oplus_{i=0}^{\infty} \mathcal{J}_{Y}^{i}\right) \times_{X} X^{\prime}=X_{Y} \times_{X} X^{\prime}$. Then the finite surjective morphism $f$ induces by base-change a finite surjective morphism $\widetilde{f}: X_{Y^{\prime}}^{\prime}=X_{Y} \times_{X} X^{\prime} \rightarrow X_{Y}$. As $\operatorname{dim}\left(X_{Y^{\prime}}^{\prime}\right)=\operatorname{dim}\left(X_{Y}\right), \widetilde{f}$ is also surjective. Clearly, $\tilde{f}^{*}(E)=E^{\prime}$, where $E^{\prime}$ is the exceptional locus of $X_{Y^{\prime}}^{\prime}$. Then for every $\eta \in \mathbb{R}$, the $\mathbb{R}$-line bundle $A+\eta E$ is ample if and only if the $\mathbb{R}$-line bundle $\tilde{f}^{*}(A+\eta E)=A^{\prime}+\eta E^{\prime}$ is ample (because $\tilde{f}$ is finite and surjective). This fact and the definition of the Seshadri constant prove then the equality $\varepsilon\left(Y^{\prime}, A^{\prime}\right)=\varepsilon(Y, A)=: \varepsilon$.

Now by (7), for every $\eta \in(0, \varepsilon) \cap \mathbb{Q}$ we have

$$
\delta_{\eta}(Y, A)=-\sum_{t=0}^{k-2}\left(\begin{array}{c}
k-2 \\
t
\end{array}\right) \eta^{t} \int_{Y} s_{y-k+t+2}(N) \cdot c_{1}\left(A_{Y}\right)^{\cdot(k-t-2)}
$$

and

$$
\delta_{\eta}\left(Y^{\prime}, A^{\prime}\right)=-\sum_{t=0}^{k-2}\left(\begin{array}{c}
k-2 \\
t
\end{array}\right) \eta^{t} \int_{Y^{\prime}} s_{y-k+t+2}\left(N^{\prime}\right) \cdot c_{1}\left(A_{Y^{\prime}}^{\prime}\right)^{\cdot(k-t-2)},
$$

where $N$ and $N^{\prime}$ are the normal bundles of $Y$ in $X$ and of $Y^{\prime}$ in $X^{\prime}$ respectively. If we set $g:=f \mid Y^{\prime}: Y^{\prime} \rightarrow Y$, then we have $N^{\prime}=g^{*}(N)$, whence $s_{y-k+t+2}\left(N^{\prime}\right)=g^{*}\left(s_{y-k+t+2}(N)\right)$ for every $t=0,1, \ldots, k-t-2$. Since the morphism $g$ is flat (as a finite surjective morphism between smooth varieties), by [12, Proposition 3.1, (d), p. 48], we get

$$
\begin{aligned}
& \int_{Y^{\prime}} s_{y-k+t+2}\left(N^{\prime}\right) \cdot c_{1}\left(A_{Y^{\prime}}^{\prime}\right)^{\cdot(k-t-2)}=\int_{Y^{\prime}} g^{*}\left(s_{y-k+t+2}(N)\right) \cdot c_{1}\left(g^{*}\left(A_{Y}\right)\right)^{\cdot k-t-2}= \\
= & \int_{Y^{\prime}} g^{*}\left(s_{y-k+t+2}(N) \cdot c_{1}\left(A_{Y}\right)^{\cdot(k-t-2)}\right)=\operatorname{deg}(g) \int_{Y} s_{y-k+t+2}(N) \cdot c_{1}\left(A_{Y}\right)^{\cdot(k-t-2),}
\end{aligned}
$$

for every $t=0,1, \ldots, k-2$. Substituting in (48) and (49) and using the obvious equality $\operatorname{deg}(f)=\operatorname{deg}(g)$, we get $\delta_{\eta}\left(Y^{\prime}, A^{\prime}\right)=\operatorname{deg}(f) \delta_{\eta}(Y, A)$. This concludes the proof of the proposition.

Remark 3.17 If in Proposition 3.16 we take $X=\mathbb{P}^{k}$ then, by Fulton-Hansen connectedness theorem (see [13]), $f^{-1}(Y)$ is always connected.

Example 3.18 To illustrate the use of Proposition 3.16 let $Y$ be the Veronese surface in $X=\mathbb{P}^{5}$. By Proposition 3.10, $Y$ is Seshadri $\mathcal{O}_{\mathbb{P}^{5}}(1)$-big. Fix an integer $n \geq 2$, and let $H_{n}$ be a smooth hypersurface of $\mathbb{P}^{5}$ that intersects $Y$ transversely (i.e., such that $C_{n}:=H_{n} \cap Y$ is a smooth curve). Consider the cyclic covering $f: X^{\prime} \rightarrow X$ of degree $n$ branched along $H_{n} \in\left|\mathcal{O}_{\mathbb{P} 5}(n)\right|$ and determined by $\mathcal{O}_{\mathbb{P} 5}(1)$ with $\mathcal{O}_{\mathbb{P} 5}(n)=\mathcal{O}_{\mathbb{P} 5}\left(H_{n}\right)$. As $H_{n}$ is smooth, $X^{\prime}$ is also smooth. Moreover, by [7, Lemma (17.1), p. 55], the canonical class $\omega_{X^{\prime}}$ is given by the formula

$$
\omega_{X^{\prime}}=f^{*}\left(\omega_{\mathbb{P}^{5}} \otimes \mathcal{O}_{\mathbb{P}^{5}}(n-1)\right)=f^{*}\left(\mathcal{O}_{\mathbb{P}^{5}}(n-7)\right) .
$$

It follows that $X^{\prime}$ is a Fano manifold for $n \leq 6, X^{\prime}$ has trivial canonical class for $n=7$, and the canonical class of $X^{\prime}$ is ample for $n \geq 8$. Moreover, $Y^{\prime}=f^{-1}(Y)$ is the cyclic covering $g: Y^{\prime} \rightarrow \mathbb{P}^{2}$ of degree $n$ branched along the smooth curve $C_{n} \in\left|\mathcal{O}_{Y}(n)\right|=\left|\mathcal{O}_{\mathbb{P}^{2}}(2 n)\right|$ (determined by the line bundle $\mathcal{O}_{Y}(1)=\mathcal{O}_{\mathbb{P}^{2}}(2)$ with $\left.\mathcal{O}_{Y}(n)=\mathcal{O}_{Y}\left(C_{n}\right)\right)$. As above, since $C_{n}$ is smooth, $Y^{\prime}$ is also smooth. Moreover, again by [7, Lemma (17.1), p. 55], we have

$$
\omega_{Y^{\prime}}=g^{*}\left(\omega_{Y} \otimes \mathcal{O}_{Y}(n-1)\right)=g^{*}\left(\mathcal{O}_{\mathbb{P}^{2}}(2 n-5)\right) .
$$

In particular, if $n=2, Y^{\prime}$ is a Del Pezzo surface of degree 2 (with canonical class $\omega_{Y^{\prime}}=$ $\left.g^{*}\left(\mathcal{O}_{\mathbb{P}^{2}}(-1)\right)\right)$ while, if $n \geq 3, Y^{\prime}$ is a surface of general type (with ample canonical class $\omega_{Y^{\prime}}=$ $g^{*}\left(\mathcal{O}_{\mathbb{P}^{2}}(2 n-5)\right)$. As $Y$ is Seshadri $\mathcal{O}_{\mathbb{P}^{5}}(1)$-big, $Y^{\prime}$ is Seshadri $f^{*}\left(\mathcal{O}_{\mathbb{P}^{5}}(1)\right)$-big by Proposition 3.16 . 
Remark 3.19 Corollary 5.3 of the next section will show that all the examples of Seshadri $\mathcal{O}_{\mathbb{P}^{k}}(1)$-big submanifolds of $\mathbb{P}^{k}$ given in this section are actually Seshadri $\mathcal{O}_{\mathbb{P}^{k}}(1)$-ample.

\section{Seshadri positivity and formal functions}

In this section we prove some general results which give further motivations for the study of Seshadri $A$-bigness and $A$-ampleness. Let us start showing the following fact.

Proposition 4.1 Under the above notation, if $Y$ is Seshadri A-ample in a smooth projective variety $X$ then $Y$ meets every irreducible hypersurface of $X$.

Proof. Let $D$ be a closed irreducible hypersurface of $X$, and set $D^{\prime}=\pi^{-1}(D)$. Then $D^{\prime}$ is a hypersurface of $X_{Y}$. As $\mathcal{H}$ is the intersection of general very ample divisors $H_{1}, \ldots, H_{k-2} \in$ $\left|m A^{*}-n E\right|$, the intersection $D^{\prime} \cap \mathcal{H}$ is a curve in $\mathcal{H}$. Finally, since $Y^{\prime}$ is an ample divisor on the surface $\mathcal{H}$, the intersection $Y^{\prime} \cap D^{\prime}=Y^{\prime} \cap\left(D^{\prime} \cap \mathcal{H}\right)$ is not empty, which clearly implies that $Y \cap D \neq \varnothing$.

The proof of the first general result of the paper, Theorem 4.5 below, is based upon the following lemma. To this purpose, recall that the cohomological dimension of an algebraic scheme $V$ over the field $\mathbb{C}$ of complex numbers is

$$
\operatorname{cd}(V):=\min \left\{t \mid H^{i}(V, \mathcal{F})=0 \text { for any } i>t \text { and for any coherent sheaf } \mathcal{F} \text { on } V\right\} .
$$

Lemma 4.2 Let $Y$ be a smooth $y$-dimensional subvariety of a smooth $k$-dimensional polarized variety $(X, A)$ such that $1 \leq y \leq k-1$. If $Y$ is Seshadri A-ample then $\operatorname{cd}\left(X_{Y} \backslash Y^{\prime}\right) \leq k-2$.

Proof. As usual, let $H_{1}, \ldots, H_{k-2}$ be $k-2$ general members of $\left|m A^{*}-n E\right|$, with $m, n$ big enough (so that $m A^{*}-n E$ is very ample). As above, set $\mathcal{H}:=H_{1} \cap \cdots \cap H_{k-2}$ and $Y^{\prime}:=\mathcal{H} \cap E$. For every $i=0, \ldots, k-3$, set $U_{i}:=\left(H_{1} \cap \cdots \cap H_{k-2-i}\right) \backslash Y^{\prime}$ and set $U_{k-2}:=X_{Y} \backslash Y^{\prime}$. Then the closure $\bar{U}_{i}$ of $U_{i}$ is $\mathcal{H}_{i}:=H_{1} \cap \cdots \cap H_{k-2-i}$ and $\bar{U}_{k-2}=X_{Y}$. It follows that $\bar{U}_{i}$ is a smooth connected projective variety of dimension $i+2$ for every $0 \leq i \leq k-2$, because $H_{1}, \ldots, H_{k-2} \in\left|m A^{*}-n E\right|$

are general. Moreover, every $\bar{U}_{i}$ contains $Y^{\prime}$ and $U_{i-1}=U_{i} \cap H_{k-i-1}, 1 \leq i \leq k-2$. In other words $U_{i-1}$ is a hyperplane section of the (open) variety $U_{i}$. Then the conclusion is a consequence of the following

Claim $4.3 \operatorname{cd}\left(U_{i}\right) \leq i$ for every $0 \leq i \leq k-2$.

To prove the claim we proceed by induction on $i$. For $i=0, U_{0}=\mathcal{H} \backslash Y^{\prime}$, and since $Y$ is $A$-ample, $Y^{\prime}$ is an ample effective divisor on $\mathcal{H}$, whence $U_{0}$ is affine and hence $\operatorname{cd}\left(U_{0}\right)=0$ by a well known affinity criterion of Serre.

Assume therefore $1 \leq i \leq k-2$ and set $U:=U_{i}, V:=U_{i-1}$ and $H:=H_{k-1-i} \mid U$. Then $\bar{U}=\mathcal{H}_{i}, \bar{V}=\mathcal{H}_{i-1}, H_{k-1-i}$ is a very ample divisor on $\bar{U}$, and hence $H$ is a very ample divisor on $U$. By induction's hypothesis we may assume $\mathrm{cd}(V) \leq i-1$, and then we have to prove that $\operatorname{cd}(U) \leq i$. To do this, for every $p \in \mathbb{Z}$ consider the cohomology exact sequence

$$
H^{i}\left(V, \mathcal{O}_{V}(p H)\right) \rightarrow H^{i+1}\left(U, \mathcal{O}_{U}((p-1) H)\right) \stackrel{\alpha_{p}}{\rightarrow} H^{i+1}\left(U, \mathcal{O}_{U}(p H)\right) \rightarrow H^{i+1}\left(V, \mathcal{O}_{V}(p H)\right)
$$

in which the first and the last spaces are zero because $\operatorname{cd}(V) \leq i-1$. Therefore the maps $\alpha_{p}$ are isomorphisms for all $p \in \mathbb{Z}$. Then by [22, Proposition 3.1, p. 92], the inequality $\operatorname{cd}(U) \leq i$ is equivalent to the vanishings $H^{i+1}\left(U, \mathcal{O}_{U}(p H)\right)=0$ for $p \ll 0$, or else, by using the isomorphisms $\alpha_{p}$, to the vanishings $H^{i+1}\left(U, \mathcal{O}_{U}(p H)\right)=0$ for $p \gg 0$. To prove these latter vanishings consider the exact sequence of local cohomology

$$
H^{i+1}\left(\bar{U}, \mathcal{O}_{\bar{U}}\left(p H_{k-1-i}\right)\right) \rightarrow H^{i+1}\left(U, \mathcal{O}_{U}(p H)\right) \rightarrow H_{Y^{\prime}}^{i+2}\left(\bar{U}, \mathcal{O}_{\bar{U}}\left(p H_{k-1-i}\right)\right) .
$$


The first space is zero for $p \gg 0$ by Serre's theorem, so that it will be enough to show that the last space is also zero for $p \gg 0$. By using formal duality [22, Theorem 3.3, p. 94] and the fact that $\operatorname{dim}(\bar{U})=i+2$, we get

$$
H_{Y^{\prime}}^{i+2}\left(\bar{U}, \mathcal{O}_{\bar{U}}\left(p H^{\prime}\right)\right)^{*} \cong H^{0}\left(\bar{U}_{/ Y^{\prime}},\left[\omega_{\bar{U}}\left(-p H^{\prime}\right)\right]_{/ Y^{\prime}}\right),
$$

where $H^{\prime}:=H_{k-1-i}, \omega_{\bar{U}}\left(-p H^{\prime}\right)=\omega_{\bar{U}} \otimes \mathcal{O}_{\bar{U}}\left(-p H^{\prime}\right)$, and $\mathcal{F}_{/ Y^{\prime}}$ denotes the formal completion along $Y^{\prime}$ of a sheaf $\mathcal{F}$ in $\bar{U}$. So everything reduces to show that the latter formal cohomology space vanishes for $p \gg 0$.

First we observe that the normal bundle $N_{Y^{\prime} \mid \bar{U}}$ of $Y^{\prime}$ in $\bar{U}$ is of the form

$$
N_{Y^{\prime} \mid \bar{U}} \cong M \oplus L^{\oplus i},
$$

where $M=N_{Y^{\prime} \mid \mathcal{H}}$ and $L=\mathcal{O}_{Y^{\prime}}\left(m A^{*}-n E\right)$. As the divisor $m A^{*}-n E$ is very ample, the line bundle $L$ is very ample. Moreover, $M$ is ample because by hypothesis $Y$ is $A$-ample. To prove (50), it is enough to observe that in $\bar{U}=H_{1} \cap \cdots \cap H_{k-2-i}$, the curve $Y^{\prime}$ is the proper intersection of $\mathcal{H}$ with $\bar{E}:=E \cap \bar{U}$, whence

$$
N_{Y^{\prime} \mid \bar{U}} \cong N_{Y^{\prime} \mid \mathcal{H}} \oplus N_{Y^{\prime} \mid \bar{E}}=M \oplus N_{\mathcal{H} \mid \bar{U}} \mid Y^{\prime} \cong M \oplus L^{\oplus i} .
$$

Now, for every $\ell \geq 0$, let $Y_{\ell}^{\prime}=\left(Y^{\prime}, \mathcal{O}_{\bar{U}} / \mathcal{J}_{Y^{\prime}}^{\ell+1}\right)$ be the $\ell$-th infinitesimal neighbourhood of $Y^{\prime}$ in $\bar{U}$, where $\mathcal{J}_{Y^{\prime}}$ is the ideal sheaf of $Y^{\prime}$ in $\bar{U}$. Then one has

$$
H^{0}\left(\bar{U}_{/ Y^{\prime}},\left[\omega_{\bar{U}}\left(-p H^{\prime}\right)\right]_{/ Y^{\prime}}\right)={\underset{\swarrow}{\ell}}_{\ell} H^{0}\left(Y_{\ell}^{\prime}, \omega_{\bar{U}} \otimes \mathcal{O}_{\bar{U}}\left(-p H^{\prime}\right) \mid Y_{\ell}^{\prime}\right) .
$$

Moreover the standard exact sequence

$$
0 \rightarrow \mathcal{J}_{Y^{\prime}}^{\ell+1} / \mathcal{J}_{Y^{\prime}}^{\ell+2} \cong \mathbf{S}^{\ell+1}\left(N_{Y^{\prime} \mid \bar{U}}^{\vee}\right) \rightarrow \mathcal{O}_{Y_{\ell+1}^{\prime}} \rightarrow \mathcal{O}_{Y_{\ell}^{\prime}} \rightarrow 0
$$

shows that for every $\ell \geq 0$ the kernel of the map

$$
\varepsilon_{\ell}: H^{0}\left(Y_{\ell+1}^{\prime}, \omega_{\bar{U}}\left(-p H^{\prime}\right) \mid Y_{\ell+1}^{\prime}\right) \rightarrow H^{0}\left(Y_{\ell}^{\prime}, \omega_{\bar{U}}\left(-p H^{\prime}\right) \mid Y_{\ell}^{\prime}\right)
$$

is $H^{0}\left(Y^{\prime}, \mathbf{S}^{\ell+1}\left(N_{Y^{\prime} \mid \bar{U}}^{\vee}\right) \otimes \omega_{\bar{U}}\left(-p H^{\prime}\right) \mid Y^{\prime}\right)$. We claim that

$$
H^{0}\left(Y^{\prime}, \mathbf{S}^{\ell+1}\left(N_{Y^{\prime} \mid \bar{U}}^{\vee}\right) \otimes \omega_{\bar{U}}\left(-p H^{\prime}\right) \mid Y^{\prime}\right)=0 \text {, for all } p \gg 0 \text { and all } \ell \geq 0 .
$$

Indeed, choose an integer $p_{0}>0$ such that $\omega_{\bar{U}}\left(-p H^{\prime}\right) \mid Y^{\prime}$ is ample for every $p \geq p_{0}$. Since $\mathbf{S}^{\ell+1}\left(N_{Y^{\prime} \mid \bar{U}}^{\vee}\right) \otimes \omega_{\bar{U}}\left(-p H^{\prime}\right) \mid Y^{\prime}$ is a direct sum of bundles of the form $M^{-\alpha} \otimes L^{-\beta} \otimes \omega_{\bar{U}}\left(-p H^{\prime}\right) \mid Y^{\prime}$ (whose inverses are ample) we have

$$
H^{0}\left(Y^{\prime}, M^{-\alpha} \otimes L^{-\beta} \otimes \omega_{\bar{U}}\left(-p H^{\prime}\right) \mid Y^{\prime}\right)=0, \text { for every } p \geq p_{0},
$$

which proves the claimed assertion (52). It thus follows that the maps $\varepsilon_{\ell}$ of the projective system are injective for every $\ell \geq 0$ and $p \geq p_{0}$. Then from (51) it follows that

$$
H^{0}\left(\bar{U}_{/ Y^{\prime}},\left[\omega_{\bar{U}}\left(-p H^{\prime}\right)\right]_{/ Y^{\prime}}\right) \cong H^{0}\left(Y^{\prime}, \omega_{\bar{U}}\left(-p H^{\prime}\right) \mid Y^{\prime}\right) \text {, for all } p \geq p_{0} .
$$

In particular, the vanishing of $H^{0}\left(\bar{U}_{/ Y^{\prime}},\left[\omega_{\bar{U}}\left(-p H^{\prime}\right)\right]_{/ Y^{\prime}}\right)$ for $p \geq p_{0}$ is equivalent to the vanishing of $H^{0}\left(Y^{\prime}, \omega_{\bar{U}}\left(-p H^{\prime}\right) \mid Y^{\prime}\right)$ for $p \geq p_{0}$. Finally, since $\operatorname{dim}\left(Y^{\prime}\right)=1>0$, the latter vanishing is obvious, so that Claim 4.3 is proved. This completes the proof of Lemma 4.2 .

Theorem 4.5 below will show that the Seshadri $A$-bigness and the Seshadri $A$-ampleness of $Y \subset X$ are related to properties of formal rational functions along $Y$ and to the cohomological dimension of the complement $U:=X \backslash Y$. First recall some definitions we need. We refer to 22. Chapters III, V], or also to [4], for more details. 
Definition 4.4 Let $V$ be an integral algebraic scheme over $\mathbb{C}, Y \subset V$ a closed subscheme, $V_{/ Y}$ the formal completion of $V$ along $Y$, and let $K\left(V_{/ Y}\right)$ be the ring of the formal rational functions of $V$ along $Y$ (see [24], or also [22]). Then $K\left(V_{/ Y}\right)$ is a field if $V$ is non-singular and $Y$ is connected (loc. cit.). Thus in this case there is a natural homomorphism of $\mathbb{C}$-algebras $K(V) \rightarrow K\left(V_{/ Y}\right)$ making $K\left(V_{/ Y}\right)$ a field extension of $K(V)$. According to 24] we say that

i) $Y$ is G2 in $V$ if the map $K(V) \rightarrow K\left(V_{/ Y}\right)$ makes $K\left(V_{/ Y}\right)$ a finite field extension of $K(V)$;

ii) $Y$ is G3 in $V$ if the map $K(V) \rightarrow K\left(V_{/ Y}\right)$ is an isomorphism.

Theorem 4.5 Let $Y$ be a smooth subvariety of dimension $y$ of a smooth complex projective polarized variety $(X, A)$ of dimension $k$ such that $1 \leq y \leq k-1$. Then the following statements hold:

1. If $Y$ is Seshadri A-big then $Y$ is $\mathrm{G} 2$ in $X$ and $k-y-1 \leq \operatorname{cd}(X \backslash Y) \leq k-1$.

2. If $Y$ is Seshadri A-ample then $Y$ is G3 in $X$ and $k-y-1 \leq \operatorname{cd}(X \backslash Y) \leq k-2$.

Proof. Under the usual notation, let $Y^{\prime}:=E \cap \mathcal{H}$, where $\mathcal{H}:=H_{1} \cap \cdots \cap H_{k-2}$ is the intersection of $k-2$ general very ample divisors $H_{1}, \ldots, H_{k-2} \in\left|m A^{*}-n E\right|$. As the intersection $Y^{\prime}=E \cap \mathcal{H}$ is proper and $N_{\mathcal{H} \mid X_{Y}} \mid Y^{\prime} \cong N_{Y^{\prime} \mid E}$, the standard exact sequence of normal bundles

$$
0 \rightarrow N_{Y^{\prime} \mid \mathcal{H}} \rightarrow N_{Y^{\prime} \mid X_{Y}} \rightarrow N_{\mathcal{H} \mid X_{Y}} \mid Y^{\prime} \rightarrow 0
$$

splits to give

$$
N_{Y^{\prime} \mid X_{Y}} \cong N_{Y^{\prime} \mid \mathcal{H}} \oplus N_{Y^{\prime} \mid E}
$$

The normal bundle $N_{Y^{\prime} \mid \mathcal{H}}$ is ample by definition of Seshadri $A$-bigness. On the other hand $N_{Y^{\prime} \mid E}$ is also ample since $Y^{\prime}$ is a complete intersection in $E$. It follows that $N_{Y^{\prime} \mid X_{Y}}$ is ample. From this and a result of Hartshorne (see [22, p. 198], or also [21, §6]) it follows that $Y^{\prime}$ is G2 in $X_{Y}$.

Now, as the morphism $\pi$ is proper and birational and $E=\pi^{-1}(Y)$, by a theorem of Hironaka and Matsumura 24] we get

$$
K\left(X_{/ Y}\right) \cong K\left(X_{Y / E}\right)
$$

where $X_{/ Y}$ (respectively $X_{Y / E}$ ) denotes the formal completion of $X$ (respectively of $X_{Y}$ ) along $Y$ (respectively along $E$ ). Since $Y^{\prime} \subset E$, we get natural maps

$$
K\left(X_{Y}\right) \rightarrow K\left(X_{Y / E}\right) \rightarrow K\left(X_{Y / Y^{\prime}}\right),
$$

where $X_{Y / Y^{\prime}}$ denotes the formal completion of $X_{Y}$ along $Y^{\prime}$. The fact that $Y^{\prime}$ is G2 in $X_{Y}$ means that $K\left(X_{Y / Y^{\prime}}\right)$ is a finite field extension of $K(X)=K\left(X_{Y}\right)$ (and in particular, $K\left(X_{Y / Y^{\prime}}\right)$ is a field). On the other hand, $K\left(X_{Y / E}\right)$ is a field because $X_{Y}$ is smooth and $E$ is connected. In particular, the second map is injective, and so $K\left(X_{Y / E}\right) \cong K\left(X_{/ Y}\right)$ is a subfield of $K\left(X_{Y / Y^{\prime}}\right)$, whence a finite field extension of $K\left(X_{Y}\right) \cong K(X)$. In other words, $Y$ is G2 in $X$.

To prove 1) observe that, since $\operatorname{dim}(Y)=y$ and $\operatorname{dim}(X)=k$, a general complete intersection of $X$ of codimension $k-y-1$ does not meet $Y$ and hence $\operatorname{cd}(X \backslash Y) \geq k-y-1$. Moreover, as $Y \neq \varnothing$, a theorem of Hartshorne and Lichtenbaum (see [21] or [22, p. 98]) implies that $\operatorname{cd}(X \backslash Y) \leq k-1$. So the first statement is proved.

To prove 2), assume that $Y$ is $A$-ample. Then, by Lemma 4.2 $\operatorname{cd}\left(X_{Y} \backslash Y^{\prime}\right) \leq k-2$ and, since $Y^{\prime}$ is G2 in $X_{Y}$, a result of Speiser applies to say that $Y^{\prime}$ is in fact G3 in $X_{Y}$, i.e., the field extension $K\left(X_{Y}\right) \subseteq K\left(X_{Y / Y^{\prime}}\right)$ is an isomorphism (see [32, or also [22, Corollary 2.2, p. 202]). As $Y^{\prime} \subset E, K\left(X_{Y / E}\right)$ is a subfield extension of $K\left(X_{Y / Y^{\prime}}\right)$, whence the field extension $K(X)=K\left(X_{Y}\right) \subseteq K\left(X_{Y / E}\right)=K\left(X_{/ Y}\right)$ is also an isomorphism, i.e., $Y$ is G3 in $X$. Since $Y$ meets every hypersurface of $X$ by Proposition 4.1 and since $Y$ is G3 in $X$, we finally get $\operatorname{cd}(X \backslash Y) \leq k-2$ by Speiser's result again. Thus part 2) also holds, and this completes the proof. 


\section{Comparing Seshadri bigness and Seshadri ampleness}

Now we come up to the second general main result of this paper (which generalizes Theorem 3.1 of [5] to the case when $Y$ is a submanifold of dimension $\geq 2$ ). Note that Theorem 5.1 below is new even in the case when $\operatorname{codim}_{X}(Y)=1$. We keep the notation as in Section 1

Theorem 5.1 Let $(X, A)$ be a polarized manifold of dimension $k \geq 3$, and let $Y$ be a submanifold in $X$ of dimension $y \geq 1$ which is Seshadri A-big. Then either $Y$ is A-ample, or there exists an irreducible hypersurface $D$ of $X$ such that $Y \cap D=\varnothing$. In the latter case the set of all irreducible hypersurfaces $D$ of $X$ such that $Y \cap D=\varnothing$ is finite.

Proof. By the $A$-bigness assumption on $Y$, we can fix an $\eta=\frac{n}{m} \in(0, \varepsilon(Y, A)) \cap \mathbb{Q}$ such that $\delta_{\eta}(Y, A)>0$, and we may assume the linear system $\left|m A^{*}-n E\right|$ to be very ample. Let $i: X_{Y} \hookrightarrow P:=\mathbb{P}\left(H^{0}\left(\mathcal{O}_{X_{Y}}\left(m A^{*}-n E\right)\right)^{\vee}\right)$ be the corresponding closed embedding, and let $L$ be a general $(k-1)$-codimensional linear subspace of $P$. Then the linear system of all $(k-2)$-codimensional linear subspaces of $P$ containing $L$ is parameterized by the $(k-2)$ dimensional projective space $\mathbb{P}^{k-2}$. For every $t \in \mathbb{P}^{k-2}$, denote by $\mathcal{H}_{t}^{\prime}$ the $(k-2)$-codimensional linear subspace of $P$ containing $L$ and corresponding to $t$, and let $\Lambda:=\left\{\mathcal{H}_{t}^{\prime}\right\}_{t \in \mathbb{P}^{k-2}}$. Setting $\mathcal{H}_{t}:=\mathcal{H}_{t}^{\prime} \cap X_{Y}$, consider the incidence correspondence of the family $\Lambda$,

$$
V^{\prime}:=\left\{(t, x) \in \mathbb{P}^{k-2} \times X_{Y} \mid x \in \mathcal{H}_{t}\right\},
$$

and denote by $p: V^{\prime} \rightarrow \mathbb{P}^{k-2}$ the restriction of the first projection of $\mathbb{P}^{k-2} \times X_{Y}$, and by $\varphi: V^{\prime} \rightarrow X_{Y}$ the restriction of the second projection. Note that, by construction, $\varphi$ coincides with the blowing up of $X_{Y}$ along $L \cap X_{Y}=\mathcal{H}_{\alpha} \cap \mathcal{H}_{\beta}$, where $\alpha$ and $\beta$ are any two different points of $\mathbb{P}^{k-2}$. In particular $V^{\prime}$ is smooth because $X_{Y}$ is smooth and the linear subspace $L$ is general (and so, by Bertini's theorem, $L \cap X_{Y}$ is smooth). Let $W^{\prime}$ be the proper inverse image of $E$ under $\varphi$, i.e., the blowing up of $E$ along $L \cap E$. Since the linear subspace $L$ is general, again by Bertini's theorem, $E \cap L$ is a finite set of reduced points. In particular, $W^{\prime}$ is also smooth because $E$ is so. Then we get the commutative diagram

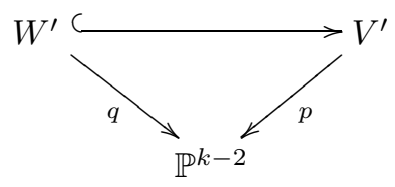

with $p$ and $q$ proper morphisms. Note that for every $t \in \mathbb{P}^{k-2}, p^{-1}(t) \cong \mathcal{H}_{t}$ and $q^{-1}(t) \cong E \cap \mathcal{H}_{t}$ under $\varphi$. Set $Y_{t}:=q^{-1}(t)=E \cap \mathcal{H}_{t}$.

We claim that the morphisms $p$ and $q$ are both flat. Indeed they are proper surjective morphisms between smooth varieties and their fibers have constant dimension (2 and 1, respectively). Then the assertion follows from a well known criterion of flatness due to Grothendieck, see [19, or also [1, $\mathrm{V},(3.5)]$.

As $Y$ is Seshadri $A$-big and $L$ is general, the normal bundle of $q^{-1}(t)=Y_{t}$ in $p^{-1}(t)=\mathcal{H}_{t}$ is ample for $t \in \mathbb{P}^{k-2}$ general. Set

$$
B:=\left\{t \in \mathbb{P}^{k-2} \mid N_{Y_{t} \mid \mathcal{H}_{t}} \text { is ample }\right\}, \quad V:=p^{-1}(B) \text { and } W:=q^{-1}(B) .
$$

Since the ampleness of $N_{Y_{t} \mid \mathcal{H}_{t}}$ is an open condition (see [17, III), $B$ is an open non-void subset of $\mathbb{P}^{k-2}$ and we get the commutative diagram

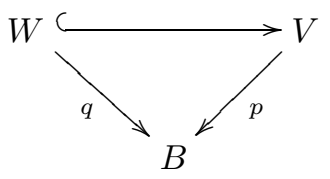


(where, by a slight abuse of notation, the restrictions $q \mid W$ and $p \mid V$ are still denoted by $q$ and $p$ respectively). Note that, since $p$ is flat, $N_{W \mid V} \mid Y_{t} \cong N_{Y_{t} \mid \mathcal{H}_{t}}$ for every $t \in B$. Therefore $N_{W \mid V}$ is a $q$-ample line bundle where, as usual, $N_{W \mid V}$ denotes the normal bundle of $W$ in $V$.

Claim 5.2 There exists a commutative diagram

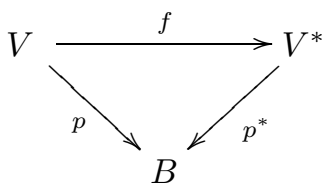

where $V^{*}$ is a normal variety, $p^{*}$ is a proper surjective morphism, and $f$ is a proper birational morphism which is an isomorphism in a neighbourhood of $W$ (and, in particular, $W$ is embedded in $V^{*}$ as a Cartier divisor) such that $\mathcal{O}_{V^{*}}(W)$ is $p^{*}$-ample.

The claim is proved in [22, III, Theorem 4.2] in the case when $B$ is a point, and extended to the general case in 22. Note that in 2] the assumption that $V$ and $W$ were projective played no role; the only thing used in the proof was that the morphisms $p$ and $q$ were proper. Note also (see 2]) that the morphism $f$ is gotten in the following canonical way. One checks first that for $r \gg 0$ the natural map $p^{*} p_{*}\left(\mathcal{O}_{V}(r W)\right) \rightarrow \mathcal{O}_{V}(r W)$ is surjective. Therefore for $r \gg 0$ there is a unique $B$-morphism $g: V \rightarrow \mathbb{P}\left(p_{*}\left(\mathcal{O}_{V}(r W)\right)\right)$ and one shows that one can take as $f$ the morphism with connected fibers arising from the Stein factorization of $g$.

Since $V^{*}$ is normal the general fiber of $p^{*}$ is also normal. Therefore, shrinking $B$ if necessary, we may assume that all the fibers of $p^{*}$ are normal. It follows that for every $t \in B$ the restriction $f_{t}:=f \mid p^{-1}(t): \mathcal{H}_{t} \rightarrow p^{*-1}(t)$ is obtained by taking the Stein factorization of the morphism defined by $\left|\mathcal{O}_{X_{Y}}(r E) \otimes \mathcal{O}_{\mathcal{H}_{t}}\right|$ for $r \gg 0$, so in particular $f_{t}$ depends only on $\mathcal{H}_{t}$, and not on the choice of the linear subspace $L$ of codimension $k-1$ contained in $\mathcal{H}_{t}^{\prime}$.

Let $Z$ (respectively $Z_{t}$ ) be the locus of $V$ (respectively of $p^{-1}(t)=\mathcal{H}_{t}$ ) consisting of all points at which the morphism $f$ (respectively $f_{t}$ ) is not a biregular isomorphism. Then it is easy to see that $Z \cap p^{-1}(t)=Z_{t}$ for every $t \in B$.

Now assume that $Y$ is not $A$-ample. Then using the above observations it follows easily that there exists an irreducible component $Z^{*}$ of $Z$ such that $\operatorname{dim}\left(Z^{*} \cap p^{-1}(t)\right) \geq 1$ for every $t \in B$, whence

$$
\operatorname{dim}\left(Z^{*} \cap p^{-1}(t)\right)=1 \text { for every } t \in B,
$$

because $p$ is of relative dimension 2 . As $\operatorname{dim}(B)=k-2$, we have $\operatorname{dim}\left(Z^{*}\right)=k-1$. Let $Z^{\prime}$ be the closure of $Z^{*}$ in $V^{\prime}$. We claim that

$$
\operatorname{dim}\left(\pi\left(\varphi\left(Z^{\prime}\right)\right)=k-1 .\right.
$$

To prove (54), recall that by Claim 5.2 the morphism $f$ is an isomorphism in a neighbourhood of $W$, whence $W \cap Z^{*}=\varnothing$. It thus follows that $Y_{t} \cap Z^{\prime}=Y_{t} \cap Z^{*}=\varnothing$ for all $t \in B$. Therefore $Z^{\prime}$ could intersect $W^{\prime}$ at most at points belonging to the union of all fibers $q^{-1}(t)=Y_{t}$ with $t \in \mathbb{P}^{k-2} \backslash B$. In particular, $Z^{\prime}$ is not contained in $W^{\prime}$, and hence $\varphi\left(Z^{\prime}\right)$ is not contained in $E$. Therefore, to prove (54), it will be enough to show that $\operatorname{dim}\left(\varphi\left(Z^{\prime}\right)\right)=k-1$ (because $\pi$ is an isomorphism off $E$ ).

Assume that this last equality does not hold, i.e., $\operatorname{dim}\left(\varphi\left(Z^{\prime}\right)\right)<k-1$. Recalling that $\varphi$ is the blowing up of $X_{Y}$ along $L \cap X_{Y}=\mathcal{H}_{\alpha} \cap \mathcal{H}_{\beta}$, with two fixed different points $\alpha, \beta \in B$, it follows that $\varphi\left(Z^{\prime}\right)=L \cap X_{Y}$. As noted above, the morphism $f_{\alpha}=f \mid \mathcal{H}_{\alpha}$ does not change if we replace the linear subspace $L$ of $P$ by another $(k-1)$-codimensional linear subspace $M$ of $P$ $(L \neq M)$ of the form $M=\mathcal{H}_{\alpha} \cap \mathcal{H}^{\prime \prime}$, where $\mathcal{H}^{\prime \prime}$ is a $(k-2)$-codimensional linear subspace of $P$, which does not belong to the family $\Lambda=\left\{\mathcal{H}_{t}^{\prime}\right\}_{t \in \mathbb{P}^{k-2}}$. In other words, $f \mid \mathcal{H}_{\alpha}$ does not change if we replace the family $\Lambda$ by a different family $\Omega=\left\{\mathcal{K}_{s}^{\prime}\right\}_{s \in \mathbb{P}^{k-2}}$ of $(k-1)$-codimensional linear 
subspaces of $P$, such that $\mathcal{H}_{\alpha}^{\prime}=\mathcal{K}_{\gamma}^{\prime}$ for some $\gamma \in \mathbb{P}^{k-2}$. Thus we can assume that there is an irreducible curve $C$ of $\mathcal{H}_{\alpha}$ contracted to a point by $f_{\alpha}=f \mid \mathcal{H}_{\alpha}$ and which is not contained in $M \cap X_{Y}$. In other words, varying $L$ a bit, we can assume that $\varphi\left(Z^{\prime}\right)$ is not contained in $L \cap X_{Y}$, which proves equality (54).

To prove the first part of the theorem, it is sufficient to show that

$$
\varphi\left(Z^{\prime}\right) \cap E=\varnothing .
$$

Indeed, from (54) and (55) it follows that $D:=\pi\left(\varphi\left(Z^{\prime}\right)\right)$ is a hypersurface of $X$ such that $D \cap Y=\varnothing$.

Assuming that (55) fails to be true, pick a point $x \in \varphi\left(Z^{\prime}\right) \cap E$. Then, since $X_{Y}$ is smooth and recalling equality (54), we have

$$
\operatorname{dim}\left(\varphi\left(Z^{\prime}\right) \cap E\right) \geq \operatorname{dim}\left(\varphi\left(Z^{\prime}\right)\right)+\operatorname{dim}(E)-\operatorname{dim}\left(X_{Y}\right)=\operatorname{dim}\left(\varphi\left(Z^{\prime}\right)\right)-1=k-2 .
$$

Therefore there is an irreducible subvariety $\Gamma \subseteq \varphi^{-1}\left(Z^{\prime}\right) \cap E$ of dimension $k-2$ passing through $x$. As $\mathcal{H}_{t}$ is a $(k-2)$-dimensional complete intersection in $X_{Y}, \mathcal{H}_{t} \cap \Gamma \neq \varnothing$ for every $t \in \mathbb{P}^{k-2}$. In particular, for every $t \in B$,

$$
\left(Z^{*} \cap W\right) \cap p^{-1}(t)=\left(Z^{\prime} \cap W^{\prime}\right) \cap p^{-1}(t) \neq \varnothing,
$$

which contradicts the fact that $Z^{*} \cap W=\varnothing$. This proves (55), and thereby the first part of the theorem.

To prove the second part of the statement, let $D$ be a hypersurface of $X$ such that $Y \cap D=\varnothing$. Set $D^{\prime}:=\pi^{-1}(D)$. Then $D^{\prime} \cap E=\varnothing$. Let $D^{*}$ be the proper transform of $D^{\prime}$ via $\varphi: V^{\prime} \rightarrow X_{Y}$. If $D^{*} \cap V=\varnothing$ then $p\left(D^{*}\right) \subseteq \mathbb{P}^{k-2} \backslash B$, whence $\operatorname{dim}\left(p\left(D^{*}\right)\right) \leq k-3$. On the other hand since $\operatorname{dim}\left(D^{*}\right)=k-1$ and $p$ is of relative dimension 2 , one gets $\operatorname{dim}\left(p\left(D^{*}\right)\right) \geq k-3$, whence $\operatorname{dim}\left(p\left(D^{*}\right)\right)=k-3$. It follows that $D^{*}$ is an irreducible component of the algebraic subset $p^{-1}\left(\mathbb{P}^{k-2} \backslash B\right)$ of $V$, so that there are finitely many possibilities for $D^{*}$ (and hence also for $D$ ) if $D^{*} \cap V=\varnothing$. Assume now that $D^{*} \cap V \neq \varnothing$. As $D^{\prime} \cap E=\varnothing$, we get $D^{*} \cap W^{\prime}=\varnothing$, whence $\widetilde{D}:=D^{*} \cap V$ does not meet $W$. Since $\operatorname{dim}(\widetilde{D})=k-1, \widetilde{D} \cap p^{-1}(t)$ is a curve on $p^{-1}(t)=\mathcal{H}_{t}$ which does not meet $Y_{t}$ for all $t \in B$. Since $f_{t}$ is given on $p^{-1}(t)$ by the linear system $\left|r Y_{t}\right|, r \gg 0$, it follows that $\widetilde{D} \cap p^{-1}(t) \subseteq Z_{t}$ for every $t \in B$, whence $\widetilde{D} \subseteq Z$. If fact, for dimension reasons, $\widetilde{D}$ is an irreducible component of $Z$, and there are only finitely many irreducible components of $Z$. This completes the proof of the theorem.

Corollary 5.3 Let $X$ be a $k$-dimensional projective manifold, $k \geq 3$, polarized by an ample line bundle $A$. Let $Y$ be a smooth subvariety of $X$ of dimension $y \geq 1$. Then the following statements hold:

1. $Y$ is Seshadri A-ample if and only if $Y$ is Seshadri A-big and $\operatorname{cd}(X \backslash Y) \leq k-2$.

2. $Y$ is Seshadri A-ample if and only if $Y$ is Seshadri A-big and $Y$ meets every hypersurface $D$ in $X$.

In particular, for $X=\mathbb{P}^{k}$, Seshadri $\mathcal{O}_{\mathbb{P}^{k}}(1)$-bigness is equivalent to is Seshadri $\mathcal{O}_{\mathbb{P}^{k}}(1)$ ampleness.

Proof. It follows immediately from Proposition 4.1. Theorem 4.5 and Theorem 5.1.

Corollary 5.4 Let $Y$ be a submanifold of codimension one of a polarized manifold $(X, A)$ which is Seshadri A-big (e.g. if the normal bundle $N$ of $Y$ in $X$ satisfies the condition (21) of Corollary [2.3). Then the set of irreducible hypersurfaces of $X$ that do not meet $Y$ is finite. 
Proof. Everything follows from Corollary 2.3 and Theorem 5.1 .

Example 5.5 Let $Y$ be a smooth subvariety of $X=\mathbb{P}^{k}$ of dimension $y \geq 1$. By Corollary 5.3. $Y$ is $\mathcal{O}_{\mathbb{P} k}(1)$-ample if and only if it is Seshadri $\mathcal{O}_{\mathbb{P}^{k}}(1)$-big. Now, assume $Y$ is $\mathcal{O}_{\mathbb{P} k}(1)$-ample in $\mathbb{P}^{k}$, and let $x \in \mathbb{P}^{k} \backslash Y$. Let $u: X^{\prime} \rightarrow \mathbb{P}^{k}$ be the blowing up of $\mathbb{P}^{k}$ at $x$, and denote by $F=u^{-1}(x)$ the exceptional locus of $X^{\prime}$. Then the closed embedding $i: Y \hookrightarrow \mathbb{P}^{k}$ lifts to a (unique) closed embedding $Y \hookrightarrow X^{\prime}$. As the line bundle $\mathcal{O}_{X^{\prime}}(-F)$ is $u$-ample, there is a sufficiently big $n \in \mathbb{N}$ such that $A:=u^{*}\left(\mathcal{O}_{\mathbb{P}^{k}}(n)\right) \otimes \mathcal{O}_{X^{\prime}}(-F)$ is ample on $X^{\prime}$. Since $Y \cap F=\varnothing$, $A_{Y} \cong u^{*}\left(\mathcal{O}_{\mathbb{P}^{k}}(n)\right) \mid Y \cong \mathcal{O}_{Y}(n)$. Then we claim that $Y$ is Seshadri $A$-big, but not $A$-ample. Indeed, the fact that $Y$ is not $A$-ample follows from Corollary 5.3(2), because $Y \cap F=\varnothing$. On the other hand, since $Y$ is Seshadri $\mathcal{O}_{\mathbb{P}^{k}}(1)$-big in $\mathbb{P}^{k}$, by Remark 1.3 (and especially by (9) ), $Y$ is also Seshadri $\mathcal{O}_{\mathbb{P}^{k}}(n)$-big in $\mathbb{P}^{k}$, i.e.,

$$
\delta_{\eta}\left(Y, \mathcal{O}_{\mathbb{P}^{k}}(n)\right)=-\sum_{t=0}^{k-2}\left(\begin{array}{c}
k-2 \\
t
\end{array}\right) \eta^{t} \int_{Y} s_{y-k+t+2}\left(N_{Y \mid \mathbb{P}^{k}}\right) \cdot c_{1}\left(\mathcal{O}_{Y}(n)\right)^{\cdot(k-t-2)}>0,
$$

for some $\eta \in\left(0, \varepsilon\left(Y, \mathcal{O}_{\mathbb{P}^{k}}(n)\right) \cap \mathbb{Q}\right.$. Since $Y \cap F=\varnothing$ and $N_{Y \mid X^{\prime}}=N_{Y \mid \mathbb{P}^{k}}$, we also have, by (56),

$$
\begin{aligned}
\delta_{\eta}(Y, A) & =-\sum_{t=0}^{k-2}\left(\begin{array}{c}
k-2 \\
t
\end{array}\right) \eta^{t} \int_{Y} s_{y-k+t+2}\left(N_{Y \mid X^{\prime}}\right) \cdot c_{1}\left(A_{Y}\right)^{\cdot(k-t-2)} \\
& =-\sum_{t=0}^{k-2}\left(\begin{array}{c}
k-2 \\
t
\end{array}\right) \eta^{t} \int_{Y} s_{y-k+t+2}\left(N_{Y \mid \mathbb{P}^{k}}\right) \cdot c_{1}\left(\mathcal{O}_{Y}(n)\right)^{\cdot(k-t-2)} \\
& =\delta_{\eta}\left(Y, \mathcal{O}_{\mathbb{P}^{k}}(n)\right)>0 .
\end{aligned}
$$

Moreover, we could blow up a smooth subvariety of $\mathbb{P}^{k}$ which does not intersect $Y$ (instead a point $x \in \mathbb{P}^{k} \backslash Y$ ), and the same conclusion as above would be true.

Example 5.5] suggests that the (finitely many) irreducible hypersurfaces of $X$ that do not intersect a given Seshadri $A$-big submanifold $Y$ of $X$ should be rather "special". The next result gives some evidence in this sense.

Theorem 5.6 Let $(X, A)$ be a smooth projective variety of dimension $k \geq 3$ polarized by an ample line bundle $A$, and let $Y$ be a smooth closed subvariety of $X$ of dimension $y \geq 1$ which is Seshadri A-big. Let $Z$ be an irreducible hypersurface of $X$ which is a local complete intersection in $X$. If $Y$ is Seshadri A-big and the normal bundle $N_{Z \mid X}$ of $Z$ in $X$ is ample, then $Y \cap Z \neq \varnothing$.

Proof. Because the normal bundle $N_{Z \mid X}$ is ample, by [22, Theorem 4.2, p. 110] there exists a birational morphism $\varphi: X \rightarrow X_{1}$ onto a normal projective variety $X_{1}$, which is a biregular isomorphism between an open neighbourhood of $Z$ in $X$ and an open neighbourhood of $\varphi(Y)$ in $X_{1}$, such that $\varphi(Z)$ becomes an ample effective divisor on $X_{1}$. As $Y$ is Seshadri $A$-big, by Theorem 4.5 (1), $Y$ is G2 in $X$. Then by an observation of Hironaka and Matsumura (24, top of p. 65]) $\operatorname{dim} \varphi(Y)>0$. Finally, as $\varphi(Z)$ is an effective ample divisor on $X_{1}, \varphi(Y) \cap \varphi(Z) \neq \varnothing$, which, by Zariski's Connectedness Theorem, see [17, III, Théorème (4.3.1), p. 130], implies that $Y \cap Z \neq \varnothing$.

The last result shows that Seshadri $A$-ampleness and Seshadri $A$-bigness are open properties under smooth deformations. We omit the proof since it is almost identical to that of [5, Theorem 4.1]. 
Theorem 5.7 Let $X$ be a smooth projective variety and let $f: X \rightarrow B$ be a smooth proper morphism of relative dimension $k \geq 3$ onto an irreducible algebraic variety $B$ of positive dimension. Let $Y$ be a closed subvariety of $X$ such that the restriction $g:=f \mid Y: Y \rightarrow B$ is a smooth morphism of relative dimension $y \geq 1$. Let $A$ be an $f$-ample line bundle on $X$. For any $t \in B$ set $X_{t}:=f^{-1}(t), Y_{t}:=g^{-1}(t)$ and $A_{t}:=A \mid X_{t}$. Assume that there exists a point $t_{0} \in B$ such that $Y_{t_{0}}$ is Seshadri $A_{t_{0}}$-ample (respectively Seshadri $A_{t_{0}}$-big). Then there exists an open set $U$ in $B, t_{0} \in U$, such that $Y_{t}$ is Seshadri $A_{t}$-ample (respectively Seshadri $A_{t}$-big) for any $t \in U$.

Examples 5.8 Let us explicitly compute the cohomolgical dimension in some of the examples discussed in Section 3 ,

i) For a surface $Y$ in a polarized manifold $(X, A)$ which is Seshadri $A$-ample, Theorem $4.5(2)$, asserts that $k-3 \leq \operatorname{cd}(X \backslash Y) \leq k-2$. For example, let $Y$ be an irregular surface of $X=\mathbb{P}^{k}$ which is $A$-ample. E.g., take $Y=C \times \mathbb{P}^{1}$ as in Proposition 3.15 and apply Corollary 5.3 above. Then a result of Hartshorne (see [21, Theorem 8.5]) implies that $\operatorname{cd}\left(\mathbb{P}^{k} \backslash Y\right)=k-2$. On the other hand, by combining Proposition 3.8 and Corollary 5.3 we know that the rational normal scroll $\mathbb{F}_{e}$ in $\mathbb{P}^{e+3}$, with $e=1,2,3,4$, is $\mathcal{O}_{\mathbb{P}^{e+3}}(1)$-ample. By a result of Verdi 33 (see also 6, Corollary 4.2]), $\mathbb{F}_{e}$ is a set-theoretic complete intersection in $\mathbb{P}^{e+3}$. This implies that $\mathbb{P}^{e+3} \backslash \mathbb{F}_{e}$ is covered by $k-2=e+1$ affine open subsets of $\mathbb{P}^{e+3}$, whence $\operatorname{cd}\left(\mathbb{P}^{e+3} \backslash \mathbb{F}_{e}\right) \leq e=k-3$. Then $\operatorname{cd}\left(\mathbb{P}^{e+3} \backslash \mathbb{F}_{e}\right)=e$ by statement 2) of Theorem 4.5. This shows that the bounds given in Theorem 4.5 (2), are in general the best possible.

ii) Let us compute the cohomological dimension of the complement of $\mathbb{P}^{2} \times \mathbb{P}^{1}$ in $\mathbb{P}^{5}$ via the Segre embedding. By Proposition 3.12 and Corollary $5.3(2), \mathbb{P}^{2} \times \mathbb{P}^{1}$ is $\mathcal{O}_{\mathbb{P} 5}(1)$-ample. Therefore Theorem 4.5 (2), predicts that $1 \leq \operatorname{cd}\left(\mathbb{P}^{5} \backslash\left(\mathbb{P}^{2} \times \mathbb{P}^{1}\right)\right) \leq 3$. If $\operatorname{cd}\left(\mathbb{P}^{5} \backslash\left(\mathbb{P}^{2} \times \mathbb{P}^{1}\right)\right)=1$ then by [22, Corollary 2.4, p. 229] it would follow that

$$
H^{2}\left(\left(\mathbb{P}^{5}\right)^{\text {an }}, \mathbb{C}\right) \cong H^{2}\left(\left(\mathbb{P}^{2} \times \mathbb{P}^{1}\right)^{\text {an }}, \mathbb{C}\right),
$$

and this is impossible because the first space is 1-dimensional while the second is 2-dimensional. On the other hand, as it is well known, $\mathbb{P}^{2} \times \mathbb{P}^{1}$ is given in $\mathbb{P}^{5}$ by the vanishing of the $(2 \times 2)$ minors of the matrix

$$
\left(\begin{array}{lll}
x_{0} & x_{2} & x_{4} \\
x_{1} & x_{3} & x_{5}
\end{array}\right)
$$

Hence $\mathbb{P}^{5} \backslash\left(\mathbb{P}^{2} \times \mathbb{P}^{1}\right)$ is covered by three affine open subsets, so that $\operatorname{cd}\left(\mathbb{P}^{5} \backslash\left(\mathbb{P}^{2} \times \mathbb{P}^{1}\right)\right) \leq 2$. We thus conclude that $\operatorname{cd}\left(\mathbb{P}^{5} \backslash\left(\mathbb{P}^{2} \times \mathbb{P}^{1}\right)\right)=2$.

iii) In the case of the Segre embedding $i: \mathbb{P}^{3} \times \mathbb{P}^{1} \hookrightarrow \mathbb{P}^{7}$, from Proposition 3.12 and Corollary 5.3 (2), it follows that $\mathbb{P}^{3} \times \mathbb{P}^{1}$ is $\mathcal{O}_{\mathbb{P}^{7}}(1)$-ample. Therefore Theorem 4.5(2), predicts that $2 \leq$ $\operatorname{cd}\left(\mathbb{P}^{7} \backslash\left(\mathbb{P}^{3} \times \mathbb{P}^{1}\right)\right) \leq 5$. If $\operatorname{cd}\left(\mathbb{P}^{7} \backslash\left(\mathbb{P}^{3} \times \mathbb{P}^{1}\right)\right) \leq 3$, using again [22, Corollary 2.4, p. 229] we deduce that

$$
H^{2}\left(\left(\mathbb{P}^{7}\right)^{\text {an }}, \mathbb{C}\right) \cong H^{2}\left(\left(\mathbb{P}^{3} \times \mathbb{P}^{1}\right)^{\text {an }}, \mathbb{C}\right),
$$

and this is again absurd. Therefore $4 \leq \operatorname{cd}\left(\mathbb{P}^{7} \backslash\left(\mathbb{P}^{3} \times \mathbb{P}^{1}\right)\right) \leq 5$. On the other hand, as $\mathbb{P}^{3} \times \mathbb{P}^{1}$ is a rational normal scroll in $\mathbb{P}^{7}$, by $[6$, Theorem 4.1$], \mathbb{P}^{3} \times \mathbb{P}^{1}$ is defined set-theoretically by five equations in $\mathbb{P}^{7}$. This implies that $\operatorname{cd}\left(\mathbb{P}^{7} \backslash\left(\mathbb{P}^{3} \times \mathbb{P}^{1}\right)\right) \leq 4$, whence we finally get $\operatorname{cd}\left(\mathbb{P}^{7} \backslash\left(\mathbb{P}^{3} \times \mathbb{P}^{1}\right)\right)=4$.

Remark 5.9 In Section 2 we have seen that the normal bundle of a Seshadri $A$-big submanifold of codimension one in a polarized manifold of dimension $\geq 3$ need not be ample. However, Paoletti proved in 28, that the normal bundle of every Seshadri $A$-big smooth curve in a polarized threefold $(X, A)$ is ample. It would be interesting to see whether this result can be somehow generalized. For instance, is the normal bundle of every Seshadri $A$-big smooth curve in a polarized manifold $(X, A)$ of arbitrary dimension $\geq 4$ always ample? 


\section{References}

[1] A. Altman and S. Kleiman, Introduction to Grothendieck Duality Theory, Lecture Notes in Math. 146, (1970), Springer-Verlag, New York, 1970.

[2] L. Bădescu, Infinitesimal deformations of negative weights and hyperplane sections, in Algebraic Geometry, Proceedings L'Aquila 1988, Lecture Notes in Math. 1417 (1990), Springer-Verlag, $1-22$.

[3] L. Bădescu, Algebraic Surfaces, Universitext (translated by V. Masȩk), Springer-Verlag, New York-Berlin-Heidelberg, 2001.

[4] L. Bădescu, Projective Geometry and Formal Geometry, Monografie Matematyczne, Instytut Matematyczny PAN, vol. 65, New Series, Birkhäuser, 2004.

[5] L. Bădescu, M.C. Beltrametti and P. Francia, Positive curves in polarized manifolds, Manuscripta Math. 92 (1997), 369-388.

[6] L. Bădescu and G. Valla, Grothendieck-Lefschetz theory, set-theoretic complete intersections and rational normal scrolls, Journal of Algebra 324 (2010), 1636-1655.

[7] W. Barth, K. Hulek, C.A.M. Peters, and A. Van de Ven, Compact Complex Surfaces, Ergebnisse der Math. vol. 4, Springer-Verlag, Berlin, 2004.

[8] M.C. Beltrametti and A.J. Sommese, Remarks on numerically positive and big line bundles, in Projective Geometry with Applications, (ed. by E. Ballico), Lecture Notes in Pure and Applied Math. 166 (1994), 9-18, M. Dekker, New York.

[9] M.C. Beltrametti and A.J. Sommese, Notes on embeddings of blowups, Journal of Algebra 186 (1996), 861-871.

[10] S. Bloch and D. Gieseker, The positivity of the Chern classes of an ample vector bundle, Invent. Math. 12 (1971), 112-117.

[11] M. Coppens, Embedding of blowing-ups, Università degli Studi di Bologna, Dipartimento di Matematica, Seminari di Geometria 1991-1993, 89-100.

[12] W. Fulton, Intersection Theory, Ergebnisse der Math. Grenzgeb. (3) 2, Springer-Verlag, Berlin 1984.

[13] W. Fulton and J. Hanssen, A connectedness theorem for proper varieties with applications to intersections and singularities, Annals of Math. 110 (1979), 159-166.

[14] W. Fulton and R. Lazarsfeld, On the connectedness of degeneracy loci and special divisors, Acta Math. 146 (1981), 271-283.

[15] W. Fulton and R. Lazarsfeld, Positivity and excess intersection, in Enumerative and Classical Algebraic Geometry, Nice 1981, Progr. Math. 24, Birkhauser (1982), 97-105.

[16] Ph. Griffiths and J. Harris, Principles of Algebraic Geometry, John Wiley \& Sons, New York, Chichester, Brisbane, Toronto, 1978.

[17] A. Grothendieck, Éléments de Géométrie Algébrique, II, III, Première Partie, Publ. Math. IHES 8, 11 (1961), 20 (1964).

[18] A. Grothendieck, Local Cohomology, Lecture Notes in Math. 41, Springer-Verlag, NewYork, 1967.

[19] A. Grothendieck, Revêtements Étales et Groupe Fondamental, (SGA I), Lectures Notes in Math. 224, Springer-Verlag, New York, 1971.

[20] R. Hartshorne, Ample vector bundles, Publ. Math. Inst. Hautes Études Sci. no. 29 (1966), 63-94.

[21] R. Hartshorne, Cohomological dimension of algebraic varieties, Ann. of Math. 88 (1968), 403-450.

[22] R. Hartshorne, Ample Subvarieties of Ample Varieties, Lecture Notes in Math. 156, SpringerVerlag, New York, 1970.

[23] R. Hartshorne, Algebraic Geometry, GTM 52, Springer-Verlag, New York, 1977.

[24] H. Hironaka and H. Matsumura, Formal functions and formal embeddings, J. Math. Soc. Japan 20 (1986), 52-82. 
[25] S.L. Kleiman, Ample vector bundles on algebraic surfaces, Proc. Amer. Math. Soc. 21 (1969), 673-676.

[26] R. Lazarsfeld, Some applications of the theory of positive vector bundles, in Complete Intersections (Acireale, 1983, ed. by S. Greco and R. Strano), Lecture Notes in Math. 1092, SpringerVerlag (1984), 21-61.

[27] R. Lazarsfeld, Positivity in Algebraic Geometry, vols. I, II, Springer-Verlag, Berlin-HeidelbergNew York, 2004.

[28] R. Paoletti, Seshadri positive curves in a smooth projective 3-fold, Atti Accad. Naz. Lincei Rend., Matematica e Applicazioni, Ser. IX, vol. VI, (1995), 259-274.

[29] R. Paoletti, Seshadri constants, gonality of space curves and restriction of stable bundles, J. Differential Geometry 40 (1994), 475-504.

[30] A.K. Singh and U. Walther, On the arithmetic rank of certain Segre products, in Commutative Algebra and Algebraic Geometry, Contemp. Math. 390, Amer. Math. Soc., Providence, RI, 2005, pp. $147-155$.

[31] A.J. Sommese, Submanifolds of abelian varieties, Math. Ann. 233 (1978), 229-256.

[32] R. Speiser, Cohomological dimension and abelian varieties, Amer. J. Math. 95 (1973), 1-34.

[33] L. Verdi, Esempi di superficie e curve intersezioni complete insiemistiche, Boll. Unione Mat. Ital. (6) 5-A (1986), 47-53.

Lucian Bădescu and Mauro C. Beltrametti

Università degli Studi di Genova

Dipartimento di Matematica

Via Dodecaneso 35

I-16146 Genova, Italy

E-mails: badescu@dima.unige.it and beltrame@dima.unige.it 